\title{
Financial market equilibria with heterogeneous agents: CAPM and market segmentation.
}

\author{
M. Del Vigna*
}

September 12, 2011

\begin{abstract}
We consider a single-period financial market model with normally distributed returns and the presence of heterogeneous agents. Specifically, some investors are classical Expected Utility maximizers whereas some others follow Cumulative Prospect Theory. Using well-known functional forms for the preferences, we analytically prove that a Security Market Line Theorem holds. This implies that Capital Asset Pricing Model is a necessary (though not sufficient) requirement in equilibria with positive prices. We correct some erroneous results about existence of equilibria with Cumulative Prospect Theory investors which had appeared in the last few years and we give sufficient conditions for an equilibrium to exist. To circumvent the complexity arising from the interaction of heterogeneous agents, we propose a segmented-market equilibrium model where segmentation is endogenously determined.
\end{abstract}

Keywords: asset pricing, heterogeneous agents, capital asset pricing model, cumulative prospect theory.

AMS classification (2000): 91B25, 91B52, 91B69, 91G10.

JEL classification: C62, D53, G11, G12.

\section{Introduction}

Since the early works of Sharpe (1964), Lintner (1965) and Mossin (1966) on the Capital Asset Pricing Model (CAPM), several attempts have been made in order to extend this model to more general specifications of the investors' preferences. While CAPM fits well for Expected Utility (EU) maximizers, especially those who decide accordingly to the Mean-Variance (MV) principle, it is quite problematic to deal with different choice paradigms. The essence of the CAPM can be traced back to the Tobin (1958) separation principle, which in turn leads to mutual fund separation and the Security Market Line Theorem (SMLT). Once we check that a separation principle holds, i.e. when traders form their portfolios employing a restricted subset of available securities, then we are on the right way for the CAPM. Even better, if we can afford on a two-fund separation, then we will readily state that a SMLT holds. In other words, investors focus exclusively on two distinct assets; the mixture of the two gives the selected financial position.

Now, it is well-known under what conditions a two-fund separation principle holds for EU optimizers; see for example Ingersoll (1987). Briefly, we can use special forms of the utility functions and/or we can impose restrictions over the probabilistic distribution of the security payoffs. Loosely speaking, MV and normal distributions are the most synthetic, appealing and friendly ways to have a SMLT. However, if we leave the EU paradigm, fund separation and the SMLT must be proved again.

To circumvent the main difficulties, it is often assumed that the agents who people the economy are endowed with preferences that can be represented via handy functionals. Surprisingly, for particular choices of such functionals and assuming normal distributions, the MV criterion continues to hold and the CAPM can be restored. This idea is not new to the literature; see for example Barberis and Huang (2008) and De Giorgi, Hens and Levy (2011). In our work, we exploit a similar reasoning using Cumulative Prospect Theory (CPT) preferences.

In Tversky and Kahneman (1992), the authors provided the first axiomatization of CPT, an improved version of the earlier Prospect Theory (see Kahneman and Tversky (1979)) that erroneously accounted for violations of first-order stochastic dominance. The cornerstones of CPT are:

\footnotetext{
*Université Paris-Dauphine \& Università degli Studi di Pisa, matteo.delvigna@for.unipi.it.
} 
- people evaluate gains or losses, that is to say departures of the terminal wealth with respect to a reference point $(\mathrm{RP})$;

- the utility function, or value function, is concave for gains and convex for losses, implying risk aversion (seeking) in the domain of gains (losses);

- individuals are loss averse (LA); in other words, a loss hurts more than an equivalent gain;

- the decision makers assess actual probabilities correctly, but these probabilities are somehow distorted during the evaluation process; not only, the distortions on gains are generally different from those on losses.

Thus, the utility function and the distortions are complementary in determining the behavior toward risk. In particular, empirical evidence suggests that people tend to overweight relatively large gains and loss of small probabilities.

The main reason of the success of CPT is that it provides a sound alternative to EU. First, it retains a sufficient analytical tractability that allows deep mathematical investigations on portfolio optimization problems (e.g. see Bernard and Ghossoub (2010) and He and Zhou (2011) for a static version of the problem, or Jin and Zhou (2008) for a continuous time model). Secondly, it is more suitable as a descriptive theory of individual choice in risky environments, since it has been developed starting from experimental observations. However, the debate whether actual decision makers follow EU or CPT (or some other choice paradigm) is still open.

On a theoretical ground, we could split up the population of the economy in several pools and classify agents depending on the choice paradigm that suits best for each of them. In this work we consider two pools, namely that of EU maximizers and that of CPT maximizers. In what follows, we will refer to an heterogeneous agents model if traders of both groups interact, while homogeneous agents model means that only one pool is considered. In Chapman and Polkovnichenko (2009), the authors study a number of stylized one-period markets populated by heterogeneous agents endowed with the most common preference paradigms used in finance. Their main finding is that even in such a simple setting, the risk premium and the risk-free return are heavily influenced by heterogeneity. Moreover, the assumption on the presence of a representative agent in heterogeneous models is extremely misleading, since it produces qualitative and quantitative biases. For these reasons, we describe in detail the composition of our pools and our results highlight the implications of heterogeneity.

We remark that in the existing literature it is common to use the word homogeneous when referring to a single group of agents that share the same identical preferences. If those preferences have a specific functional form, it follows that the parameters used to describe them must be the same. On the other hand, heterogeneous often denotes a situation where every decision maker belongs to the same pool but the preference parameters may be different. To avoid misunderstandings, we will always specify the pool where the agents come from, EU or CPT, and whether their preferences are identical or not.

In the past few years, the analysis of existence (or non-existence) of equilibria in financial market with non-EU agents has become an important field of study. Notably, Barberis and Huang (2008) prove that CAPM necessarily arises if one assumes CPT-homogeneous agents with piecewise-power utility functions, special probability distortions and normally distributed asset payoffs. Actually, they also claim that an equilibrium with positive prices always exists. On the contrary, in this paper we will show that this result is incorrect since preference parameters can be chosen in such a way that prices become negative.

De Giorgi, Hens and Rieger (2010) show that equilibria may not exist for a pool of CPTheterogeneous traders with piecewise-power utility function and a finite number of states of nature. The main reason can be found in the discontinuity of the demand function. Besides this, if investors are able to infinitely leverage their portfolios, then we can have an ill-posed optimization problem. However, equilibrium existence is proved when the market is populated by a continuum of agents and non-negativity constraints are imposed on the terminal wealth.

De Giorgi, Hens and Levy (2011) assume normally distributed payoffs and a special form of rankdependent preferences for a single pool of heterogeneous investors. At first, the authors show that the portfolio optimization problem is equivalent to a MV one. Then, a SMLT is proved and existence of equilibria is claimed. Regarding these results, we highlight a minor flaw in the proof of an inequality that leads to erroneously accept a particular type of probability distortions. Not only, we will see once again that existence of equilibria is assured only if we admit negative prices. Actually, in De 
Giorgi, Hens and Levy (2011) the definition of market equilibrium admits non-positive prices but it seems to us that strictly positivity of prices should be a must in this type of models.

In the CAPM world, the problem of existence of equilibria and the consequent inspection of the sign of the prices are well-known; see Nielsen (1988), Allingham (1991), Nielsen (1992) and M. Levy (2007). Exactly the same questions arise in non-EU homogeneous agents models and heterogeneous agents models. To avoid further complexity, we sometimes assume the presence of a single risky asset or we focus on a special type of equilibria, namely segmented equilibria.

Market segmentation models appeared soon in financial modeling and they have been used to describe more realistic scenarios. Generally, a segmented equilibrium is the result of exogenous constraints imposed over market participants. Specifically, H. Levy (1978) considers a market where agents are limited on the number of risky assets they can use to form their portfolios. Merton (1987) assumes that each investor includes in her portfolio only a subset of securities, namely those she is informed about. Markowitz (1990) studies a CAPM where traders are constrained by a system of linear equalities and/or inequalities. In Sharpe (1991), market participants can not take negative positions in assets. However, we distinguish from the preceding literature as we provide sufficient conditions for the existence of a segmented equilibrium that arises endogenously in the model. Thus, we can classify traders in two groups, namely those who participate to the risky market and those who invest all their wealth in the risk-free asset.

As it happens in actual advanced economies, there is a surprisingly high percentage of households who stay out of the stock market. Haliassos and Bertaut (1995) use data from the 1983 Survey of Consumer Finances and note that between $75 \%$ and $80 \%$ of U.S. households do not hold risky securities directly. Looking for the reasons of this phenomenon, the authors empirically find that inertia and departures from the EU paradigm are the best explanations. In particular, rank-dependent preferences and dual theory of choice by Yaari (1987) are the tested paradigms. Bertaut (1998) still exploits data from the Survey of Consumer Finance and specifies $80 \%$ and $59 \%$ of non-participant households in 1983 and 1995 respectively, without accounting for those who hold stock indirectly. Finally, Dimmock and Kouwenberg (2010) test on the 1997-2002 DNB Household Survey (a database from a survey conducted in The Netherlands) whether LA is a crucial factor for non-participation. However, they do not confirm a significant relationship between LA and investment in equity.

We finally remark that we aim at studying theoretical properties of equilibria. We are not interested in the calibration of our models, nor we want to provide normative prescriptions.

The paper is organized as follows. In Section 2 we introduce the main assumptions of the model, namely the market structure and the preferences of the investors. Next, in Section 3 we show that CAPM is the necessary model that arises in equilibrium, even though an equilibrium needs not to exist. We analyze segmented equilibria in Section 4, also providing explicit sufficient conditions for the existence of an equilibrium. Section 5 is devoted to the study of an endogenous risk-free return model. Finally, Section 6 concludes.

\section{The model}

Consider a single-period financial market, where in $t=0$ investment decisions are made and in $t=1$ consumption of the realized profits takes place. The uncertainty underlying our market will be modeled through a given probability space $(\Omega, \mathscr{F}, \mathbb{P})$, where the random variable $Y: \Omega \rightarrow \mathbb{R}$ represents a financial position. $\mathbb{E}[Y]$ will denote the expectation of the r.v. $Y$ with respect to $\mathbb{P}$, while $\operatorname{Cov}(Y, Z)$ will be the covariance between $Y$ and $Z$.

Assumption 1 (Market structure). There are $N+1$ traded assets in a frictionless market without constraints.

- Asset 0 is the risk-free asset which pays 1 unit of currency in every state of the world; its exogenous rate of return is $r$ and it is in elastic supply.

- Assets $1, \ldots, N$ are non-redundant risky securities, whose per-share payoff is given by a multivariate normal r.v. $D=\left(D_{1}, \ldots, D_{N}\right)$, that is $D \sim N(\mu, V)$ where $\mu \in \mathbb{R}^{N}$ and $V \in \mathbb{R}^{N \times N}$ is positively defined. The exogenous supply of the risky assets is $S=\left(S_{1}, \ldots, S_{N}\right) \in \mathbb{R}_{++}^{N}{ }^{1}$.

\footnotetext{
${ }^{1} \mathbb{R}_{++}^{N}$ is short notation for $(0,+\infty)^{N}$. In this case, we mean that every asset is in strictly positive supply.
} 
We set the price of the risk-less asset as $q_{0}:=\frac{1}{1+r}$, whereas $q=\left(q_{1}, \ldots, q_{N}\right)$ will denote the prices of the $\mathrm{N}$ stocks to be determined in equilibrium. Portfolios are obtained through investment in the $N+1$ securities and as usual we can consider the market portfolio $M=D^{\top} S$, that is the sum of all available assets. Immediately, we compute the expected payoff, the variance and the price of $\mathrm{M}$ as $\mu_{M}=\mu^{\top} S, \sigma_{M}^{2}=S^{\top} V S$ and $q_{M}=q^{\top} S$ respectively.

We model heterogeneity in our economy assuming that it is populated by two distinct pools of investors, also called traders or agents. Those who belong to the first group, $\mathcal{I}$, are classical EU investors, whereas the other pool, $\mathcal{H}$, contains a special class of CPT agents. Specifically we make the following hypotheses.

Assumption 2 (Investor endowments). Each investor receives an initial wealth $W^{j}>0, j \in \mathcal{I} \cup \mathcal{H}$, in the form of traded assets.

Assumption 3 (EU preferences). $\mathcal{I}$ is the (possibly empty) set of EU traders, with $I:=\# \mathcal{I} \geq 0$. The $i$-th agent, $i=1, \ldots, I$, evaluates her random terminal wealth $X^{i}$ through

$$
U^{i}\left(X^{i}\right)=\mathbb{E}\left[u^{i}\left(X^{i}\right)\right]
$$

where $u^{i}: \mathbb{R} \rightarrow \mathbb{R}$ is a strictly increasing, strictly concave, twice differentiable utility function.

Note that Assumptions 1 and 3 taken together imply that EU investors respect the MV principle.

Assumption 4 (CPT-MV preferences). $\mathcal{H}$ is the (possibly empty) set of CPT-MV traders, with $H:=\# \mathcal{H} \geq 0$. The $h$-th agent, $h=1, \ldots, H$, evaluates her random terminal wealth $X^{h}$ through

$$
\begin{aligned}
U^{h}\left(X^{h}\right)= & \int_{R P^{h}}^{+\infty} u^{h}\left(x-R P^{h}\right) \mathrm{d}\left[-w_{+}^{h}\left(1-\mathbb{P}\left(X^{h} \leq x\right)\right)\right] \\
& +\int_{-\infty}^{R P^{h}} u^{h}\left(x-R P^{h}\right) \mathrm{d}\left[w_{-}^{h}\left(\mathbb{P}\left(X^{h} \leq x\right)\right)\right]
\end{aligned}
$$

where

(i) $u^{h}: \mathbb{R} \rightarrow \mathbb{R}$ is strictly increasing, convex on $(-\infty, 0)$, concave on $(0,+\infty)$, twice differentiable on $\mathbb{R} \backslash\{0\}$ with $u^{h}(0)=0$;

(ii) $w_{ \pm}^{h}:[0,1] \rightarrow[0,1]$ are differentiable, non-decreasing functions with $w_{ \pm}^{h}(p)=p$ for $p=0$ and $p=1$;

(iii) $R P^{h} \in \mathbb{R}$ is a deterministic reference point;

(iv) $u^{h}$ and $w_{ \pm}^{h}$ are such that $U^{h}$ is increasing in the mean and decreasing in the variance of $X^{h}$, for any $X^{h}$ such that $\mathbb{E}\left[X^{h}\right] \geq R P^{h}$.

The previous assumption captures the main points ${ }^{2}$ of CPT as originally formulated by Tversky and Kahneman (1992). In particular, (i) reflects the typical S-shaped (convex-concave) utility function. Property (ii) models the probability distortions (or weighting functions) of our investors; note that we do not restrict ourselves to reversed S-shaped (concave-convex) $w_{ \pm}^{h}$ as empirically observed in Tversky and Kahneman (1992). In (iii), we introduce the reference point $R P^{h}$ as the level which distinguishes a loss $\left(X^{h}<R P^{h}\right)$ from a gain $\left(X^{h} \geq R P^{h}\right)$. Now, in the literature there exists a variety of specifications for the reference level. In most cases, $R P^{h}$ is chosen as an exogenous deterministic benchmark; see Bernard and Ghossoub (2010) and He and Zhou (2011) among others. Alternatively, it can be represented by a stochastic wealth level as explained in Jin and Zhou (2008) or it is even determined endogenously in the model (Köszegi and Rabin (2006)). We fix $R P^{h}=W^{h}(1+r$ ); this choice is standard in equilibrium models with CPT investors (see Barberis and Huang (2008) and De Giorgi, Hens, Levy (2011) among others) but it needs an exogenous risk-free rate of return ${ }^{3}$. In

\footnotetext{
${ }^{2}$ We do not explicitly give a characterization of loss aversion. The main reason is that LA is easy to say in words ("losses loom larger than gains") but at the same time there is not a widespread accepted mathematical formulation. Common assumptions are $u^{h}(y)+u^{h}(-y)<u^{h}(x)+u^{h}(-x)$ for $y>x>0$ and $\lim _{x \rightarrow 0} u^{h^{\prime}}(|x|) / \lim _{x \rightarrow 0} u^{h^{\prime}}(-|x|)>1$, which express LA for large and small stakes respectively. For an overview on LA and its implications, we refer the reader to Schmidt and Zank (2005) and Köbberling and Wakker (2006).

${ }^{3}$ In Section 5 we will remove this assumption and the risk-less return will be determined endogenously.
} 
such a case, a return over (below) $1+r$ is regarded as a gain (loss). Finally, Property (iv) imposes a link on the choice of the utility function and the probability distortions. We remark that a negative dependence of $U^{h}$ in the variance is needed only if the expected variation of wealth is positive. In order to see that Property (iv) is not a too severe constraint, we provide the following lemma.

Lemma 1 (Cases of CPT-MV preferences). The following specifications fulfill Property (iv) of Assumption 4 .

(i)

$$
u^{h}(x)=\left\{\begin{array}{ll}
x^{\alpha^{h}} & \text { if } x \geq 0, \\
-\lambda^{h}(-x)^{\alpha^{h}} & \text { if } x<0,
\end{array} \quad w_{ \pm}^{h}(p)=\frac{p^{\delta^{h}}}{\left(p^{\delta^{h}}+(1-p)^{\delta^{h}}\right)^{1 / \delta^{h}},}\right.
$$

with $\alpha^{h} \in(0,1), \lambda^{h}>1, \delta^{h} \in(0.28,1)$.

(ii)

$$
u^{h}(x)= \begin{cases}f^{h}(x) & \text { if } x \geq 0 \\ -\lambda^{h} f^{h}(-x) & \text { if } x<0\end{cases}
$$

where $\lambda^{h}>1, f^{h}$ is non negative, strictly increasing and concave on $(0,+\infty)$ and $f^{h}(0)=0$. Moreover, $w_{-}^{h}(p)=1-w_{+}^{h}(1-p)$ and $w_{-}^{h^{\prime}}(p)<\lambda^{h} w_{-}^{h^{\prime}}(1-p)$ for all $p \in(0.5,1)$.

(iii)

$$
u^{h}(x)= \begin{cases}x & \text { if } x \geq 0 \\ \lambda^{h} x & \text { if } x<0\end{cases}
$$

where $\lambda^{h}>1$ and $w_{ \pm}^{h}(p)=p$.

Proof. (i) See Proposition A3 in the Appendix in Barberis and Huang (2008). (ii) See Proposition 2 and Lemma 1 in De Giorgi, Hens and Levy (2011). (iii) See Proposition 1 in Fortin and Hlouskova (2011).

Case ( $i$ ) is also termed Constant Relative Risk Aversion (CRRA) or piecewise-power utility. It is very close to the original formulation by Tversky and Kahneman (1992). However, the power indexes of the convex and concave parts of $u^{h}$ are assumed to be equal as well as those of the reversed Sshaped probability distortions. $\lambda^{h}>1$ models LA whereas $\delta^{h}>0.28$ ensures monotonicity of $w_{ \pm}^{h}$ (see Ingersoll (2008)). Experimental evidence in Tversky and Kahneman (1992) suggests $\alpha^{h} \approx 0.88$, $\lambda^{h} \approx 2.25, \delta^{h} \approx 0.61$ for the gains and $\delta^{h} \approx 0.69$ for the losses.

In case (ii), the assumption $w_{-}^{h}(p)=1-w_{+}^{h}(1-p)$ induces a special form of rank-dependent CPT preferences. In De Giorgi, Hens and Levy (2011), the authors focus on the CRRA case and on the piecewise-exponential utility index, where

$$
u^{h}(x)= \begin{cases}\lambda^{h,+}\left(1-\exp \left(-\alpha^{h} x\right)\right) & \text { if } x \geq 0 \\ \lambda^{h,-}\left(\exp \left(\alpha^{h} x\right)-1\right) & \text { if } x<0\end{cases}
$$

where $\alpha^{h} \in(0,1)$ and $\lambda^{h,-}>\lambda^{h,+}>0$ implies LA for large payoffs. This particular form is also studied in Köbberling and Wakker (2006) and it closely approximate the piecewise-power utility around zero for $\alpha^{h}=0.2, \lambda^{h,+}=6.52$ and $\lambda^{h,-}=14.7$, as De Giorgi, Hens and Levy (2011) show. However, these authors erroneously state that the weighting function $w$ defined in equation (3) satisfies $w^{\prime}(p)<\lambda w^{\prime}(1-p)$ for $p \in(0.5,1)$ as long as $\lambda>1$ and $\delta>0.28$. As a matter of fact, in the proof of their Lemma 2 there is a miscalculation. The correct expression should be:

$$
\begin{aligned}
z(p, \lambda, \delta) \equiv \lambda w^{\prime}(1-p)-w^{\prime}(p)=\frac{\delta\left[\lambda(1-p)^{\delta-1}-p^{\delta-1}\right]\left[p^{\delta}+(1-p)^{\delta}\right]}{\left[p^{\delta}+(1-p)^{\delta}\right]^{\frac{1}{\delta}+1}} \\
\\
-\frac{\left[\lambda(1-p)^{\delta}+p^{\delta}\right]\left[(1-p)^{\delta-1}-p^{\delta-1}\right]}{\left[p^{\delta}+(1-p)^{\delta}\right]^{\frac{1}{\delta}+1}} .
\end{aligned}
$$

For $p \in(0.5,1)$ the first addend in $(7)$ is positive, whereas the second one is negative. Hence, for suitable choices of $\lambda$ and $\delta$ the inequality $z(p, \lambda, \delta)>0$ does not hold for all $p \in(0.5,1)$, as Figure 1 shows. In the left panel we set $\lambda=2.25$ and in the right panel we fixed $\delta=0.65$. Plotting an 

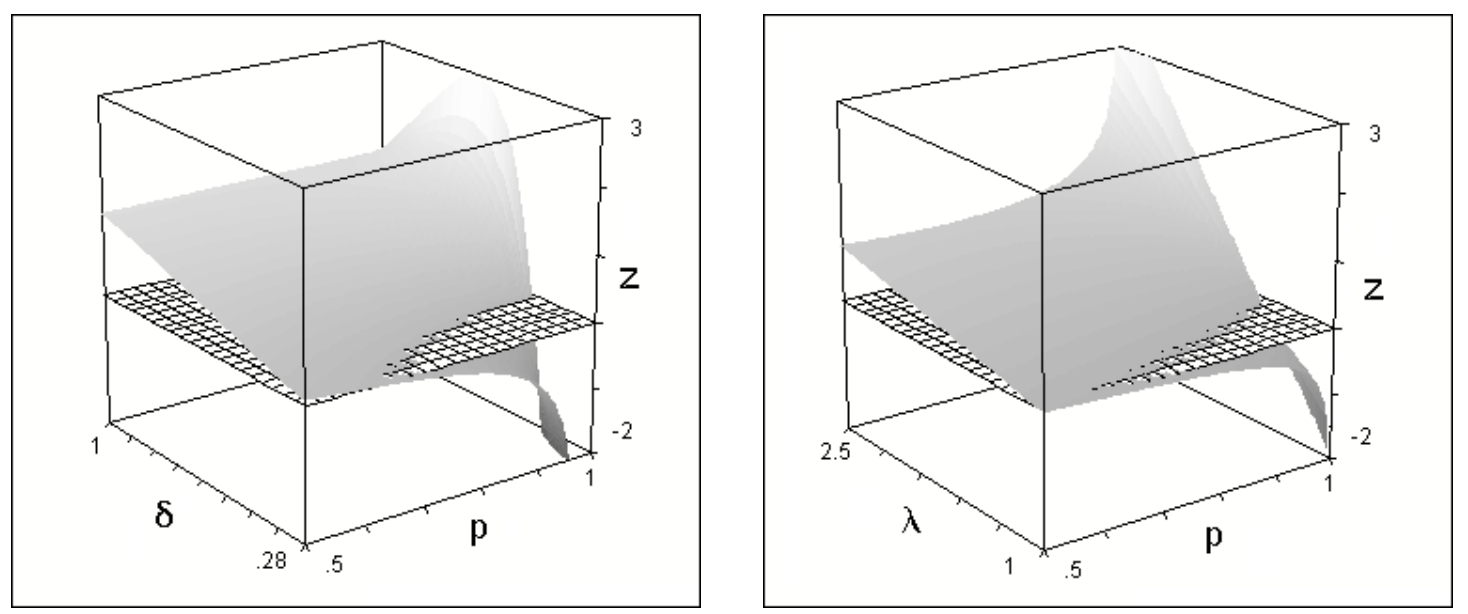

Figure 1: The figure shows that the probability distortions $w$ introduced in (3) do not always satisfy $z(p, \lambda, \delta)>0$ for $p \in(0.5,1), \lambda>1$ and $\delta \in(0.28,1)$ (the function $z$ is defined in $(7))$. In the left panel, we fixed $\lambda=2.25$ and in the right panel we fixed $\delta=0.65$. The horizontal grid is plotted at $z=0$.

horizontal grid at level $z=0$, we see that low acceptable values of $\delta$ as well as low acceptable values of $\lambda$ can cause a violation of the desired inequality.

Case (iii) is called Linear LA; note that it ignores probability distortions. Once again, $\lambda^{h}$ captures LA in its easiest (and clearest) way.

To conclude this section, we specify how our investors form their portfolios. Let $j \in \mathcal{I} \cup \mathcal{H}$ and let $\theta^{j}=\left(\theta_{1}^{j}, \ldots, \theta_{N}^{j}\right) \in \mathbb{R}^{N}$ be a portfolio, where $\theta_{n}^{j}$ is the number of shares of security $n$ held by the $j$-th trader. For a given price vector $q \in \mathbb{R}_{++}^{N}$, the budget constraint becomes $W^{j}=$ $q_{0} \theta_{0}^{j}+q^{\top} \theta^{j}$, where $\theta_{0}^{j}$ are the units of the risk-free asset acquired by agent $j$. Note that in the budget constraint we already use the equality instead of an inequality $(\leq)$ thanks to the monotonicity of the preferences with respect to the expected return. Using this constraint, we find the terminal wealth $X^{j}=W^{j}(1+r)+[D-(1+r) q]^{\top} \theta^{j}$. Recalling that a CPT-MV investor evaluates the changes in wealth, we set $\Delta X^{h}:=X^{h}-R P^{h}=[D-(1+r) q]^{\top} \theta^{h}, h=1, \ldots, H$, and we observe that $X^{j}$ as well as $\Delta X^{h}$ are scalar normal r.v. for every portfolio choice.

\section{CAPM as a necessary condition in equilibrium}

Assumptions 1 and 4-(iv) are very strong but extremely helpful in finding some necessary conditions that must hold in equilibrium. Before giving a formal definition of an equilibrium, we recall that for a MV investor the solution to her portfolio optimization problem will lie on the MV frontier. In other words, it will be a linear combination of the risk-less asset and the "tangency" portfolio". Not only, equilibrium means that the market must be cleared, hence it is necessary for the tangency portfolio to be the market portfolio $M$. Moreover, the aggregate demand for $M$ must be sufficiently high for all the shares of each security to be purchased. By itself, this fact does not imply that anyone must express positive demand for $M$. However, the economy as a whole has to re-allocate the endowments to exactly clear the market. We now use mathematics to translate these facts.

Definition 1 (Market equilibrium). An equilibrium is a vector of strictly positive prices $\bar{q} \in \mathbb{R}_{++}^{N}$ and allocations $\bar{\theta}^{j} \in \mathbb{R}^{N}, j \in \mathcal{I} \cup \mathcal{H}$, such that

(i) (Portfolio optimization) given $\bar{q}, \bar{\theta}^{j} \in \arg \max _{\theta^{j} \in \mathbb{R}^{N}} U^{j}\left(X^{j}\right), j \in \mathcal{I} \cup \mathcal{H}$;

(ii) (Market clearing) given $\bar{q}, \sum_{i=1}^{I} \bar{\theta}_{n}^{i}+\sum_{h=1}^{H} \bar{\theta}_{n}^{h}=S_{n}, n=1, \ldots, N$.

\footnotetext{
${ }^{4}$ Tangency occurs between the MV frontiers obtained with and without the risk-free asset. In the first case, it is formed by two straight lines on the mean/standard deviation plane. On the contrary, it is an hyperbola when only risky assets are available; see Ingersoll (1987).
} 
Assume for the moment that individual optimal portfolios contain the risk-free asset and the market portfolio exclusively. Therefore, to solve the $j$-th agent's optimization problem we only need to establish the proportion of the market portfolio $M$ that she is willing to acquire. Letting $\Theta_{M}^{j}$ be such proportion, we can write the terminal wealth as $X^{j}=W^{j}(1+r)+\Theta_{M}^{j}\left[M-(1+r) q_{M}\right]$ and similarly obtain the wealth changes $\Delta X^{h}$. Now it is immediate to see that (1) induces the function

$$
v^{i}\left(\Theta_{M}^{i}, q_{M}\right)=\int_{\mathbb{R}} u^{i}\left(W^{i}(1+r)+\Theta_{M}^{i}\left[x-(1+r) q_{M}\right]\right) \phi_{\mu_{M}, \sigma_{M}}(x) \mathrm{d} x,
$$

where $\phi_{\mu, \sigma}$ is the probability density function (pdf) of a $N(\mu, \sigma)$ r.v.. Performing a change of variable in (2), we also find

$$
\begin{aligned}
v^{h}\left(\Theta_{M}^{h}, q_{M}\right)= & \int_{0}^{+\infty} u^{h}(t) \mathrm{d}\left[-w_{+}^{h}\left(1-\Phi_{\Theta_{M}^{h}\left[\mu_{M}-(1+r) q_{M}\right],\left|\Theta_{M}^{h}\right| \sigma_{M}}(t)\right)\right] \\
& +\int_{-\infty}^{0} u^{h}(t) \mathrm{d}\left[w_{-}^{h}\left(\Phi_{\Theta_{M}^{h}\left[\mu_{M}-(1+r) q_{M}\right],\left|\Theta_{M}^{h}\right| \sigma_{M}}(t)\right)\right]
\end{aligned}
$$

where $\Phi_{\mu, \sigma}$ denotes the cumulative distribution function (cdf) of a $N(\mu, \sigma)$ r.v.. Note that we used the absolute value of $\Theta_{M}^{h}$ for the standard deviation in (9) because we admit $\Theta_{M}^{h} \in \mathbb{R}$, i.e. short selling and taking leverage are allowed. Considering $q_{M} \in \mathbb{R}_{+}$as a parameter, the function $v^{j}$ allows us to find the optimal demand $\Theta_{M}^{j \star}\left(q_{M}\right)$.

Lemma 2. We have $\Theta_{M}^{j \star} \geq 0$ if and only if $\frac{\mu_{M}}{q_{M}} \geq 1+r$. Moreover, inequalities are strict if $j \in \mathcal{I}$.

Proof. For an EU investor, Assumption 3 on the strict concavity of $u^{i}$ implies that utility maximization has a unique solution. From the First-Order Conditions (FOC) in equation (8), we find

$$
\frac{\partial v^{i}}{\partial \Theta_{M}^{i}}\left(0, q_{M}\right)=u^{i^{\prime}}\left(W^{i}(1+r)\right)\left[\mu_{M}-(1+r) q_{M}\right]
$$

which implies $\Theta_{M}^{i \star}\left(q_{M}\right)>0$ if and only if the term inside the brackets is positive.

For a CPT-MV agent it suffices to see that if $\mu_{M}-(1+r) q_{M} \geq 0$, then the terminal position obtained through a particular $\Theta_{M}^{h}>0$ is never worse than the final wealth reached through $-\Theta_{M}^{h}$. This is because they lead to the same variance but the first investment has a higher expected return. Hence, for any given $q_{M}$ we have $v^{h}\left(\Theta_{M}^{h}, q_{M}\right) \geq v^{h}\left(-\Theta_{M}^{h}, q_{M}\right)$ for every $\Theta_{M}^{h}>0$. A reversed relation holds if $\mu_{M}-(1+r) q_{M} \leq 0 .^{5}$

The meaning of the preceding lemma is that the optimal demand for the market portfolio is non negative if and only if the return of the market portfolio is greater than that of the risk-less asset; for the EU agents we also have strict inequalities. However, for Lemma 2 to be applied we have to prove that the market portfolio is the only risky position undertaken by our investors; but this is true as they all follow the MV criterion. Consequently, we have the following necessary condition.

Lemma 3 (Necessary condition in equilibrium). Under Assumptions 1-4, a market equilibrium in the sense of Definition 1 can exist only if $\frac{\mu_{M}}{q_{M}} \geq 1+r$.

Proof. The market clearing condition (ii) in Definition 1 becomes $\sum_{i=1}^{I} \bar{\Theta}_{M}^{i}+\sum_{h=1}^{H} \bar{\Theta}_{M}^{h}=1$. If $\mu_{M}-(1+r) q_{M}<0$ then $\Theta_{M}^{i}<0$ and $\Theta_{M}^{h} \leq 0$ for every $i=1, \ldots, I$ and $h=1, \ldots, H$, which violates the previous equality.

An immediate consequence of Lemma 3 is that the market portfolio $M$ lies on the efficient frontier. In order to recover more familiar expressions, we compute the returns of the risky securities $R:=$ $\left(R_{1}, \ldots, R_{N}\right)$, where $R_{n}=D_{n} / q_{n}$. Then, $R_{M}:=M / q_{M}$ and $R_{f}:=1+r$ represent the market return and the risk-free return respectively. Formally, we can summarize our results in a Security Market Line Theorem (SMLT), which is the essence of CAPM.

\footnotetext{
${ }^{5}$ For CPT-MV investors, a strict version of the inequalities can not be obtained since specific choices of $u^{h}$ and $w_{ \pm}^{h}$ can cause indifference between the values of $\Theta_{M}^{h}$. This happens for example in the Linear LA and in the CRRA case, as it will be shown later.
} 
Proposition 1 (Security Market Line Theorem). Under Assumptions 1-4, every equilibrium in the sense of Definition 1 satisfies

(i) $\mathbb{E}\left[R_{M}\right] \geq R_{f}$;

(ii)

$$
\mathbb{E}\left[R_{n}\right]-R_{f}=\beta_{n}\left(\mathbb{E}\left[R_{M}\right]-R_{f}\right), \quad n=1, \ldots, N
$$

where

$$
\beta_{n}=\frac{\operatorname{Cov}\left(R_{n}, R_{M}\right)}{\sigma_{M}^{2}} .
$$

Proof. Suppose an equilibrium exists. Then (i) follows immediately from Lemma 3. To obtain (ii), it suffices to note that a portfolio whose return is $R_{M}+x\left(R_{n}-R_{f}\right)$ must reach its maximum Sharpe ratio $^{6}$ at $x=0$, as suggested in Barberis and Huang (2008).

Equation (10) is the classical linear relation which synthesizes the CAPM. We highlight the fact that if an equilibrium exists, then the positive prices $\bar{q}$ give us the returns $R, R_{M}$ and we can check that (10) holds. On the contrary, if we only require (10) to hold, then it is not guaranteed the existence of an equilibrium in the sense of Definition 1. Even worse, we could be able to find optimal allocations $\theta^{j}$ which clear the market but require some negative prices. To better understand how these pathological phenomena arise, we distinguish three cases depending on the population of the market.

\subsection{Markets with EU investors}

Consider the case when our market is populated by solely $\mathrm{EU}$ agents, i.e. $\mathrm{I}>0$ and $\mathrm{H}=0$; such a situation is the most common in the literature. Problems concerning the existence of CAPM equilibria with possibly negative prices has already been studied; see Nielsen (1988), Allingham (1991), Nielsen (1992) and M. Levy (2007). Briefly, the main cause for the negative prices to arise stands in the non monotonicity of MV preferences. Holding more risky shares causes expected return and variance to rise simultaneously and the overall effect on the expected utility could be negative. Consequently, the aggregate demand is systematically lower than the exogenous supply unless prices become negative, which correspond to a sort of extreme reward for bearing risk. While the aforementioned authors analyze this issue in full generality, we restrict ourselves to the Constant Absolute Risk Aversion (CARA) case as it allows easier and explicit computations. Namely, we make the following assumption.

Assumption 5 (CARA EU preferences). For $i=1, \ldots, I, u^{i}(x)=-\exp \left(-\lambda^{i} x\right), \lambda^{i}>0$.

Now it is possible to solve the optimization problem for the $i$-th trader and then aggregate over $i$. Nielsen (1992) clearly shows in his Example 2 that the CAPM equilibrium price system is necessarily given by

$$
\bar{q}=\frac{1}{R_{f}}(\mu-\Lambda V S),
$$

where $\Lambda=\left(\sum_{i=1}^{I} \lambda^{i-1}\right)^{-1}$ is the harmonic mean of the CARA coefficients, also known as the "market price of risk". Consequently, prices will be strictly positive if and only if $\mu-\Lambda V S \in \mathbb{R}_{++}^{N}$. Observe that $\bar{q}$ is totally expressed in terms of exogenous parameters and if $\bar{q} \in \mathbb{R}_{++}^{N}$, then the induced price of the market portfolio $q_{M}$ will be positive. However, the contrary is not necessarily true, as some prices could be positive and some others negative or zero.

In the simplest case of a market with one risk-free and one risky asset, we can identify the only security with the market portfolio. The following relations hold:

$$
q_{M}=q_{1} S_{1}, \quad \mu_{M}=\mu_{1} S_{1}, \quad \sigma_{M}=\sigma_{1} S_{1}, \quad \theta_{1}^{i}=\Theta_{M}^{i} q_{M} .
$$

Hence, in equilibrium there is one price to be determined and it is easy to check whether it is positive. In particular, the FOC from equation (8) give

$$
\Theta_{M}^{i \star}\left(q_{M}\right)=\frac{\mu_{M}-R_{f} q_{M}}{\lambda^{i} \sigma_{M}^{2}},
$$

\footnotetext{
${ }^{6}$ The Sharpe ratio of a security with expected return $\mu$ and standard deviation $\sigma$ is given by $S R:=\frac{\mu-R_{f}}{\sigma}$.
} 
and summation over $i$, together with the market clearing condition, yields the equilibrium price

$$
\bar{q}_{1}=\frac{1}{R_{f}}\left(\mu_{1}-\Lambda \sigma_{1}^{2} S_{1}\right),
$$

which is positive if and only if $\Lambda<\frac{\mu_{1}}{\sigma_{1}^{2} S_{1}} \equiv \frac{\mu_{M}}{\sigma_{M}^{2}}$. In such a case, the equilibrium allocations will be $\bar{\theta}_{1}^{i}=\frac{\Lambda}{\lambda^{i}} S_{1}, i=1, \ldots, I$.

\subsection{Markets with CPT-MV investors}

Opposite to the previous case, we now assume $I=0$ and $H>0$ to have a market populated by solely CPT-MV traders. As we have just seen in Section 3.1, the MV criterion is not a sufficient condition for the existence of CAPM equilibria. Indeed, we are going to show that even in some simple examples there is not an equilibrium. Thus, our findings clash with those in Barberis and Huang (2008) and De Giorgi, Hens and Levy (2011). The reason is the same as before; even for an arbitrarily small (or zero) price, every CPT-MV will refuse to hold a positive amount of the market portfolio. As a consequence, the market clearing condition can not be attained and no equilibrium with positive prices exists. Before proving the fallacy of the existing results, we provide an illustrative example where an equilibrium can not be found.

Example 1 (No equilibrium with Linear LA agents). Consider a market with the risk-free asset and a unique risky asset with exogenous supply $S_{1}>0$ and a random per-share payoff $D_{1} \sim N\left(\mu_{1}, \sigma_{1}^{2}\right)$. The parameters that describe the preferences of our CPT-MV investors are as in Lemma 1, (iii). To fix ideas, suppose $\lambda^{1} \geq \lambda^{2} \geq \ldots \geq \lambda^{H}>1$, i.e. agent $H$ is the less loss adverse of the pool; intuitively, she is the trader mostly inclined in bearing risk. We now show that if $\lambda^{H}$ is strictly greater than a certain threshold $\tilde{\lambda}$, then the optimal demand for the risky security is zero for every trader, no matter how close to zero the price $q_{1}$ is. Thus, an equilibrium with positive prices does not exist.

Formally, the Linear LA specification in equation (9) gives

$$
v^{h}\left(\Theta_{M}^{h}, q_{M}\right)=\Theta_{M}^{h}\left[\int_{0}^{+\infty} s \phi_{\mu_{M}-R_{f} q_{M}, \sigma_{M}}(s) \mathrm{d} s+\lambda^{h} \int_{-\infty}^{0} s \phi_{\mu_{M}-R_{f} q_{M}, \sigma_{M}}(s) \mathrm{d} s\right],
$$

which is linear in $\Theta_{M}^{h}$, strictly decreasing in $\lambda^{h}$ and $q_{M}$ (recall that $\mu_{M}-R_{f} q_{M} \geq 0$ ). Therefore, it suffices to check that for $q_{M}=0^{7}$, the term inside the brackets is negative for the $H$-th trader. Computing the integrals in equation (16), we see that this is indeed true as long as

$$
\lambda^{H}>\tilde{\lambda} \equiv \frac{\sigma_{M}+\sqrt{2 \pi} \mu_{M} e^{\mu_{M}^{2} / 2 \sigma_{M}^{2} \Phi\left(\mu_{M} / \sigma_{M}\right)}}{\sigma_{M}-\sqrt{2 \pi} \mu_{M} e^{\mu_{M}^{2} / 2 \sigma_{M}^{2} \Phi\left(-\mu_{M} / \sigma_{M}\right)}}
$$

where $\Phi$ is the cumulative distribution function of a standard normal r.v.. We remark that the threshold $\tilde{\lambda}$ does not depend on $R_{f}$; using the relation $\mu_{M} / \sigma_{M}=\mu_{1} / \sigma_{1}$, we see that $\tilde{\lambda}$ is increasing in $\mu_{1}$ and decreasing in $\sigma_{1}$. Finally, if one considers the index of riskiness $\alpha:=\mu_{1} / \sigma_{1}$, then $\tilde{\lambda}$ is increasing in $\alpha$ as expected.

Inspired by the previous example, we now show that a CAPM equilibrium as claimed in Barberis and Huang (2008), Proposition 1, may fail to exist. Specifically, the authors assume a frictionless market with normally distributed payoffs and CPT-MV homogeneous investors whose preferences are as in Lemma 1, (i). Homogeneity means that every agent shares the same parameters $\alpha^{h}, \lambda^{h}$ and $\delta^{h}$ (we thus suppress the superscripts when unnecessary). Under these hypotheses, Barberis and Huang (2008) state that a CAPM equilibrium always exists and it inherits the properties listed in our Proposition 1. When proving their result in the Appendix, the authors say that they can compute the equilibrium price vector using $N-1$ non-redundant equations from (10) and an additional equation (no. (19) in their article) which yields the market price $q_{M}$. For the reader's convenience, we report here that equation:

$$
V\left(\hat{R}_{M}\right) \equiv-\int_{-\infty}^{0} w\left(P\left(\hat{R}_{M}\right)\right) \mathrm{d} u\left(\hat{R}_{M}\right)+\int_{0}^{+\infty} w\left(1-P\left(\hat{R}_{M}\right)\right) \mathrm{d} u\left(\hat{R}_{M}\right)=0,
$$

\footnotetext{
${ }^{7}$ If we use returns in our computations, then $q_{M}=0$ does not make sense. However, one could perform a limit for $q_{M} \downarrow 0$ thanks to the continuity of $v^{h}$.
} 
where $\hat{R}_{M}=R_{M}-R_{f}$ is the market portfolio excess return and $P$ is the cdf of $\Delta X^{h}$. Unfortunately, equation (18) is misleading when looking for an equilibrium because it can lead to equilibria with negative prices.

We focus on this issue in a simple market as illustrated in Example 1. The only price to be determined is the market price $q_{M}$, or equivalently $q_{1}$. Starting from equation (9), we can perform integration by parts as explained in Barberis and Huang (2008), Lemma 1, and we obtain

$$
v^{h}\left(\Theta_{M}^{h}, q_{M}\right)=\left(\Theta_{M}^{h}\right)^{\alpha} f\left(q_{M}\right),
$$

where

$$
f\left(q_{M}\right)=-\int_{-\infty}^{0} w\left(\Phi_{\mu_{M}-R_{f} q_{M}, \sigma_{M}}(s)\right) \mathrm{d} u(s)+\int_{0}^{+\infty} w\left(1-\Phi_{\mu_{M}-R_{f} q_{M}, \sigma_{M}}(s)\right) \mathrm{d} u(s) .
$$

Equation (19) gives the optimal solution to the portfolio optimization problem:

$$
\Theta_{M}^{h \star}= \begin{cases}0 & \text { if } f\left(q_{M}\right)<0, \\ \text { any } \Theta_{M}^{h} \geq 0 & \text { if } f\left(q_{M}\right)=0, \\ +\infty & \text { if } f\left(q_{M}\right)>0\end{cases}
$$

Consequently, an equilibrium exists if and only if $f$ vanishes at some $\bar{q}_{M}>0$. Note that $f$ is a continuous, monotone decreasing function and $\lim _{q_{M} \downarrow-\infty} f\left(q_{M}\right)=+\infty, \lim _{q_{M} \uparrow+\infty} f\left(q_{M}\right)=-\infty$. Hence, it always exists a unique solution $\bar{q}_{M} \in \mathbb{R}$ of equation (20). If $\bar{q}_{M}>0$, then it will be the equilibrium price and the equilibrium allocations can be arbitrarily chosen as long as they satisfy $\sum_{h=1}^{H} \bar{\Theta}_{M}^{h}=1$. Substantially, we proved the following result.

Proposition 2 (No equilibrium with CPT-MV homogeneous CRRA agents). Assume $I=0$ and $H>0$ (only CPT-MV agents). Let Assumptions 1, 2 and 4 hold with homogeneous CPT-MV preferences as in equation (3) and let $f$ be defined as in equation (20). If $f(0)<0$ then there are no financial equilibria.

A closer look to equation (20) shows that it is very similar to equation (18), but they are not the same. In fact, Barberis and Huang (2008) exploit the market portfolio return $R_{M}$ instead of the market portfolio price $q_{M}$ in order to find an equilibrium. Their procedure is not correct because $\mu_{M}$ and $\sigma_{M}^{2}$ are exogenous, whereas $q_{M}$ must be determined endogenously. Therefore, even if one is able to find a pair $\left(\frac{\mu_{M}}{q_{M}}, \frac{\sigma_{M}^{2}}{q_{M}^{2}}\right)$ that satisfies equation (18), this does not mean that there exists a $q_{M}>0$ which solves equation (20). In fact for any given $\mu_{M}$ and $\sigma_{M}$, the price $q_{M}$ influences simultaneously the mean of the market return and its variance.

In Barberis and Huang (2008), Section III.A, the authors provide a numerical example. To begin, they use the normalization $\hat{R}_{M} \sim N\left(\mu_{M}, \sigma_{M}\right)$ (see their equation no. (30)). After that, they specify the parameters as follows: $\left(\alpha, \delta, \lambda, R_{f}, \sigma_{M}\right)=(0.88,0.65,2.25,1.02,0.15)$. Inserting these values into equation (18), they correctly state that $V\left(\hat{R}_{M}\right)=0$ if $\mu_{M}=0.075$ (that is to say, a gross expected return equal to 1.095). However, they do not exhibit a specific level of the market price $q_{M}$ nor they specify the exogenous supplies $S$ and the payoffs $D$.

On the contrary, the first step of the appropriate procedure which leads to the equilibrium price $q_{M}>0$ (if it exists) consists in fixing $S_{1}, \mu_{1}$ and $\sigma_{1}$. Second, using equation (13) we can compute $\mu_{M}$ and $\sigma_{M}$ and finally we can look for a solution to equation (20). As an example, we can select $S_{1}$ and $\mu_{1}$ such that $\mu_{M}=1.095$. Leaving unchanged the other parameters, that is $\left(\alpha, \delta, \lambda, R_{f}\right)=$ $(0.88,0.65,2.25,1.02)$, we can plot $f\left(q_{M}\right)$ for different values of $\sigma_{M}$ (see Figure 2). The results are quite clear; an equilibrium exists as long as $\sigma_{M}$ lies in a suitable range (numerical simulations show $\left.\sigma_{M} \in(0,2.19)\right)$. On the contrary, for some specifications there is not a strictly positive solution to $f\left(q_{M}\right)=0$. In other words, no market price can sustain an equilibrium. A similar situation arises if we change the values of some preference parameters. In a numerical analysis, we fixed $\left(\alpha, \delta, R_{f}, \mu_{M}, \sigma_{M}\right)=(0.88,0.65,1.02,1.095,1.75)$ and allowed $\lambda$ to vary. Results are shown in Figure 3 ; in this case we can find an equilibrium only if $\lambda \in(1,2.77)$.

Now, we extend the previous analysis to more general specifications of the CPT-MV preferences. First of all, note that homogeneity is an extremely strong assumption. Allowing heterogeneity into the pool of CPT-MV traders makes our scenario a more realistic one, though it does not exclude absence of equilibria. In fact, consider a group of investors whose preferences fulfill all the requirements of 


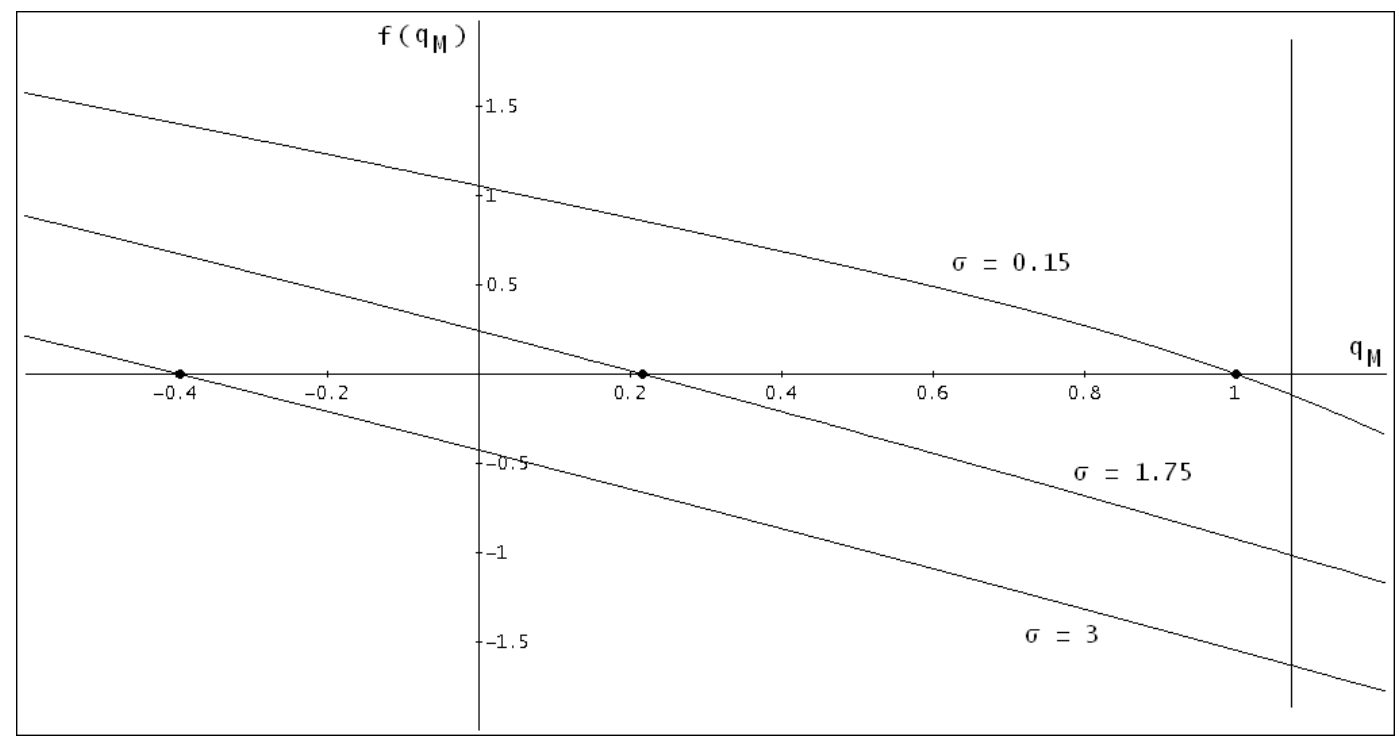

Figure 2: The figure shows three curves of the function $f\left(q_{M}\right)$ introduced in (20). The preference parameters are $(\alpha, \delta, \lambda)=(0.88,0.65,2.25)$, the market parameters are $\left(R_{f}, \mu_{M}\right)=(1.02,1.095)$ and $\sigma_{M}$ takes the values $0.15,1.75,3$. We highlighted with a dot the respective equilibrium prices: $\bar{q}_{M}=1$ (as in the example of Barberis and Huang (2008)), $\bar{q}_{M}=0.216$ and $q_{M}=-0.396$ (which must be refused). The vertical line represents the upper limit on $q_{M}$ as explained in Lemma 3.

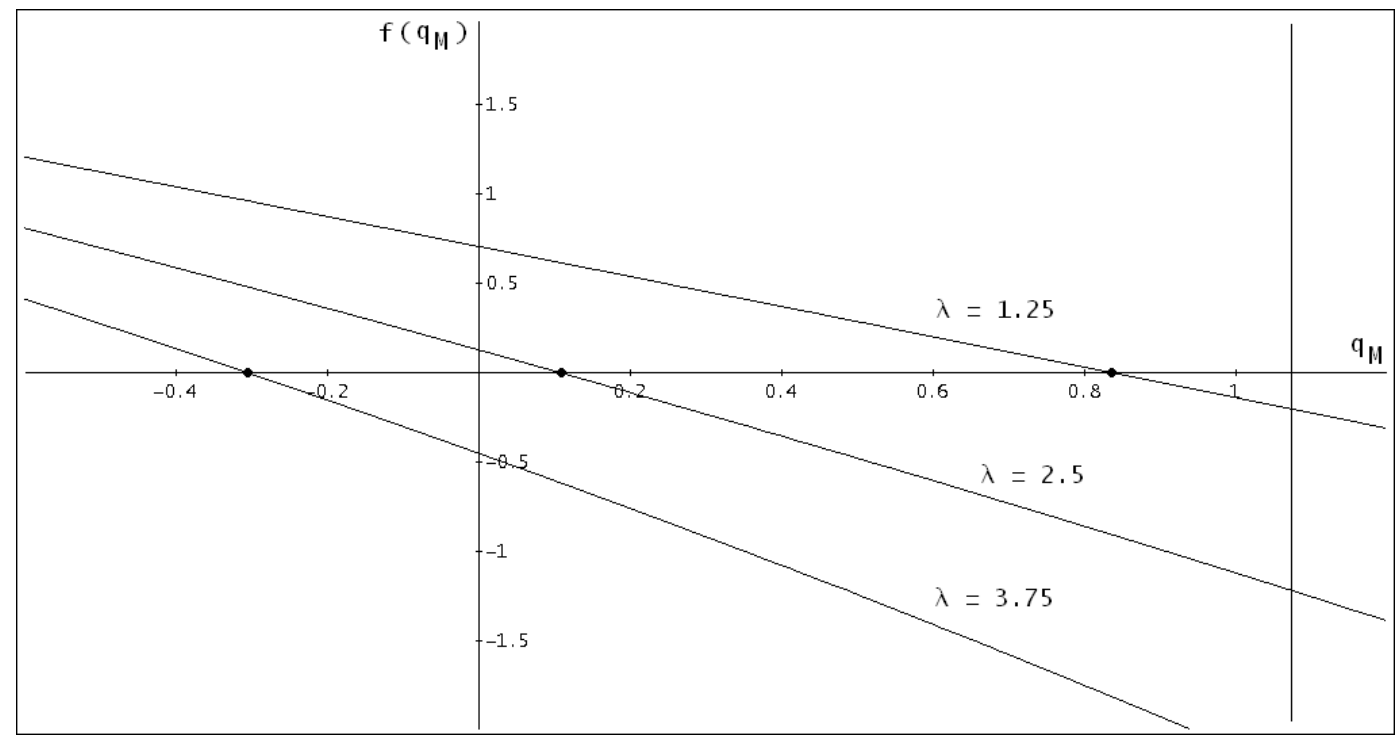

Figure 3: The figure shows three curves of the function $f\left(q_{M}\right)$ introduced in (20). The market parameters are $\left(R_{f}, \mu_{M}, \sigma_{M}\right)=(1.02,1.095,1.75)$, the preference parameters are $(\alpha, \delta)=(0.88,0.65)$ and $\lambda$ takes the values $1.25,2.5,3.75$. We highlighted with a dot the respective equilibrium prices: $\bar{q}_{M}=0.836, \bar{q}_{M}=0.107$ and $\bar{q}_{M}=-0.306$ (which must be refused). The vertical line represents the upper limit on $q_{M}$ as explained in Lemma 3.

Lemma 1, (ii), with $f^{h}(x)=x^{\alpha^{h}}$ for possibly distinct $\alpha^{h}, \lambda^{h}$ and $w_{ \pm}^{h}$. This case includes the one studied in De Giorgi, Hens and Levy (2011), Section 4.2, where it is assumed $w_{-}^{h}$ as in equation (3) (with appropriate choices of $\lambda^{h}$ and $\delta^{h}$ as explained in Section 2). For $\sigma_{M}>0$, define the Sharpe ratio of the market portfolio as

$$
\xi:=\frac{\mu_{M}-R_{f} q_{M}}{\sigma_{M}}, \quad q_{M} \in\left(0, \frac{\mu_{M}}{R_{f}}\right] .
$$

Observe that the bounds on $q_{M}$ imply $\xi \in\left[0, \frac{\mu_{M}}{\sigma_{M}}\right)$. Now we use equation (22) to compute $v^{h}$ as a 
function of $\Theta_{M}^{h}$ and $\xi$. With an abuse of notation, we write

$$
v^{h}\left(\Theta_{M}^{h}, \xi\right)=\left(\Theta_{M}^{h} \sigma_{M}\right)^{\alpha^{h}} F^{h}(\xi),
$$

where

$$
F^{h}(\xi)=\int_{-\xi}^{+\infty}(t+\xi)^{\alpha^{h}} \mathrm{~d}\left[w_{-}^{h}(\Phi(t))\right]-\lambda^{h} \int_{-\infty}^{-\xi}(-t-\xi)^{\alpha^{h}} \mathrm{~d}\left[w_{-}^{h}(\Phi(t))\right],
$$

assuming that the previous integrals exist. Our equation (24) is the analogous to equation no. (12) in De Giorgi, Hens and Levy $(2011)^{8}$. In their Lemma 2, the authors correctly prove that choosing $w_{-}^{h}$ as in $(3), F^{h}$ is continuous, strictly increasing on $\mathbb{R}_{+}$and $F^{h}(0)<0$. Moreover, they show that $\lim _{\xi \uparrow+\infty} F^{h}(\xi)=+\infty$ and they use this argument to prove the existence of an equilibrium in their Proposition 6. Unfortunately, if we look for equilibria with strictly positive prices, performing the limit for $\xi \uparrow+\infty$ does not make any sense (recall the upper bound on $\xi$ ). More properly, an equilibrium can exist if we find $\bar{\xi} \in\left[0, \frac{\mu_{M}}{\sigma_{M}}\right)$ such that

$$
F^{h}(\bar{\xi}) \leq 0 \text { for all } h=1, \ldots, H \text { and } F^{h^{\prime}}(\bar{\xi})=0 \text { for at least one } h^{\prime} .
$$

Obviously, one can compute back $\bar{q}_{M}$ using $\bar{\xi}$ and equation (22). We remark that (25) is a necessary condition but not a sufficient one as claimed in De Giorgi, Hens and Levy (2011), Proposition 6. In fact, in the presence of multiple risky assets, finding $\bar{q}_{M}>0$ does not imply a positive price for each security. On the contrary, in the simpler case of a single risky asset, $\bar{q}_{M}>0$ ensures the existence of an equilibrium. We summarize our analysis in the following proposition.

Proposition 3 (Necessary condition with CPT-MV heterogeneous CRRA utility). Assume $I=0$ and $H>0$ (only CPT-MV agents). Let Assumptions 1, 2 and 4 hold with heterogeneous preferences as in Lemma 1, (ii), with $f^{h}(x)=x^{\alpha^{h}}$ and let $F^{h}$ be defined as in equation (24). If there is a financial equilibrium, then there exists a $\bar{\xi} \in\left[0, \frac{\mu_{M}}{\sigma_{M}}\right)$ such that (25) holds.

As De Giorgi, Hens and Levy (2011) note, in this case the attainable equilibria are not economically meaningful. If condition (25) holds, it is usually satisfied for exactly one $h^{\prime}$. That is to say, due to heterogeneity of the traders' preferences it is extremely unlikely to find a $\bar{\xi}$ such that $F^{h^{\prime}}(\bar{\xi})=F^{h^{\prime \prime}}(\bar{\xi})=0$ for distinct $h^{\prime}$ and $h^{\prime \prime}$. Thus, the resulting equilibrium implies optimal null risky investment for all agents but one and this last investor clears the security market optimally acquiring the whole market portfolio. Actually, this agent is indifferent with respect to its financial position as equation (23) shows.

We now leave aside the CRRA case which causes such a highly unrealistic scenario and we focus on the piecewise-exponential utility index introduced in (6). The advantage of this specification stands in its asymptotic behavior. Ignoring the effect of the probability distortions, we see that reaching an infinite gain implies $u^{h}(x) \rightarrow \lambda^{h,+}$, whereas unbounded losses causes $u^{h}(x) \rightarrow \lambda^{h,-}$. Being $\lambda^{h,-}>\lambda^{h,+}>0$, these investors will avoid extreme leverage or short-selling and the optimal proportion $\Theta_{M}^{h}$ will be zero or a finite amount. To be more precise, in this case we have ${ }^{9}$

$$
v^{h}\left(\Theta_{M}^{h}, \xi\right)=\lambda^{h,+} \int_{-\xi}^{+\infty}\left(1-e^{-\Theta_{M}^{h} \sigma_{M}(t+\xi)}\right) \mathrm{d} w_{-}^{h}(\Phi(t))+\lambda^{h,-} \int_{-\infty}^{-\xi}\left(e^{\Theta_{M}^{h} \sigma_{M}(t+\xi)}-1\right) \mathrm{d} w_{-}^{h}(\Phi(t)) .
$$

Considering the Sharpe ratio $\xi \in\left[0, \frac{\mu_{M}}{\sigma_{M}}\right)$ as a parameter, we define the optimal demand function

$$
\Theta_{M}^{h \star}(\xi)=\arg \max _{\Theta_{M} \geq 0} v^{h}\left(\Theta_{M}, \xi\right) .
$$

In their Lemma 3, De Giorgi, Hens and Levy (2011) prove that (27) is a good definition. Moreover, they show that $\Theta_{M}^{h \star}$ is continuous in $\xi, \lim _{\xi \downarrow 0} \Theta_{M}^{h \star}(\xi)=0$ and $\lim _{\xi \uparrow+\infty} \Theta_{M}^{h \star}(\xi)=+\infty$. Again, the last limit makes sense mathematically but not economically. Hence it can not be used as an argument to prove the existence of an equilibrium (with positive prices) as De Giorgi, Hens and Levy (2011) do in their Proposition 7. Intuitively, in this case an equilibrium with positive prices can exist if we are able to find a $\xi \in\left[0, \frac{\mu_{M}}{\sigma_{M}}\right)$ such that the aggregate demand of the CPT-MV traders exceeds the aggregate supply, represented by the market portfolio. We formalize this fact in the next result.

\footnotetext{
${ }^{8}$ In De Giorgi, Hens and Levy (2011) the Sharpe ratio is denoted with $q$; here we use $q$ to denote the price vector.

${ }^{9}$ Compare with the expression of $g^{i}(q, \sigma)$ in the proof of Lemma 3 in De Giorgi, Hens and Levy (2011). In their notation, $\sigma$ represents the standard deviation of the terminal wealth, equal to our $\Theta_{M}^{h} \sigma_{M}$.
} 
Proposition 4 (Necessary condition with CPT-MV heterogeneous piecewise-exponential utility). Assume $I=0$ and $H>0$ (only CPT-MV agents). Let Assumptions 1, 2 and 4 hold with heterogeneous preferences as in Lemma 1, (ii), with $u^{h}$ defined as in (6). If there is a financial equilibrium, then there exists $\hat{\xi} \in\left[0, \frac{\mu_{M}}{\sigma_{M}}\right)$ such that

$$
\sum_{h=1}^{H} \Theta_{M}^{h \star}(\hat{\xi})>1 .
$$

Proof. For any $h, \Theta_{M}^{h \star}$ is continuous in $\xi$ and converges to zero as $\xi \downarrow 0$. These properties are inherited by the summation over $h$. Therefore, there exists a sufficiently small $\tilde{\xi} \geq 0$ such that $\sum_{h=1}^{H} \Theta_{M}^{h \star}(\tilde{\xi})<1$. By the intermediate value theorem we deduce the existence of a $\bar{\xi}$ which fulfills equality in (28).

Finally, note that (28) can not be formulated using the weak inequality $(\geq)$. In fact, suppose $\Theta_{M}^{h \star}$ are strictly monotone increasing and $\sum_{h=1}^{H} \Theta_{M}^{h \star}\left(\frac{\mu_{M}}{\sigma_{M}}\right)=1$. Then, for any $\xi<\frac{\mu_{M}}{\sigma_{M}}$ we have $\sum_{h=1}^{H} \Theta_{M}^{h \star}(\xi)<1$ and $\bar{\xi}=\frac{\mu_{M}}{\sigma_{M}}$ must be refused as it implies $q_{M}=0$.

In any case, $\bar{\xi}$ will induce the equilibrium market price through (22) and $\Theta_{M}^{h \star}(\bar{\xi})$ will be the equilibrium allocations. In the presence of $N$ risky securities one has to check that the $N$ prices are strictly positive. This can be done using $\bar{q}_{M}$ and $N-1$ non-redundant equations from (10).

\subsection{Markets with both types of investors}

Mixing the results of the previous analysis we can study more realistic markets where the two pools of traders interact. So far, we have seen that a CARA EU agent is willing to acquire risky securities and her optimal demand is a decreasing function of the market price (see equation (14)). This behavior is generally shared by the CPT-MV investors; however, the particular shape of the utility function $u^{h}$ can cause serious troubles leading to unbounded optimal positions. This feature lowers the chances of having an equilibrium and it heavily restricts the type of attainable equilibria. Loosely speaking, we could distinguish between well-behaved traders (whose demand for the stocks is bounded, e.g. CARA EU and piecewise-exponential CPT-MV investors) and ill-behaved traders (whose demand is potentially unbounded, e.g. Linear LA and piecewise-power CPT-MV investors).

When all agents are well-behaved we are able to provide sufficient conditions for the existence of a positive market price $q_{M}$, which is equivalent to the existence of an equilibrium in a market with one risky asset. In this last case, we have the following result:

Proposition 5 (Sufficient conditions for the existence of an equilibrium). Assume $I>0$ and $H>0$ (both types of agents). Let Assumptions 1-5 hold with CPT-MV heterogeneous preferences as in Lemma 1, (ii), with $u^{h}$ defined as in (6). Each of the following conditions implies the existence of an equilibrium:

(i) $\Lambda<\frac{\mu_{M}}{\sigma_{M}^{2}}$;

(ii) there exists a $\hat{\xi} \in\left[0, \frac{\mu_{M}}{\sigma_{M}}\right)$ such that

$$
\frac{1}{\Lambda \sigma_{M}} \hat{\xi}+\sum_{h=1}^{H} \Theta_{M}^{h \star}(\hat{\xi})>1
$$

Proof. First recall that for a CARA EU agent the optimal demand $\Theta_{M}^{i \star}$ is given by (14), which can be written as $\Theta_{M}^{i \star}(\xi)=\frac{1}{\lambda^{i} \sigma_{M}} \xi$. Summation over $i$ yields an aggregate demand by the EU traders, equal to $\frac{1}{\Lambda \sigma_{M}} \xi$. Now, the presence of both types of investors implies the following market clearing condition: $\frac{1}{\Lambda \sigma_{M}} \xi+\sum_{h=1}^{H} \Theta_{M}^{h \star}(\xi)=1$. Note that the left-hand side of the previous equality is a continuous function of $\xi$ which converges to zero as $\xi \downarrow 0$.

Suppose (i) holds. Then for $\xi=\frac{\mu_{M}}{\sigma_{M}}$ we have

$$
\frac{1}{\Lambda \sigma_{M}} \xi+\sum_{h=1}^{H} \Theta_{M}^{h \star}(\xi) \geq \frac{\mu_{M}}{\Lambda \sigma_{M}^{2}}>1
$$


and we conclude thanks to the intermediate value function theorem. A similar argument applies for (ii), where the strict inequality is justified as in the proof of Proposition 4.

The intuition behind the previous result is straightforward. If ( $i$ ) holds, the presence of EU CARA agents is sufficient to assure the existence of an equilibrium independently on the presence of a group of well-behaved CPT-MV investors. In any case, the pool $\mathcal{H}$ is helpful in reaching an equilibrium when $(i)$ is not fulfilled since CPT-MV traders can contribute to clear the risky market.

At this point, adding ill-behaved agents potentially damages our results. Even the presence of a unique ill-behaved agent could be extremely dangerous, as long as her optimal policy is to exploit infinite leverage on some securities. On the contrary, if such an ill-behaved trader stays out of the stock market, then her impact on the equilibrium prices becomes irrelevant ${ }^{10}$. We are going to exploit this idea in a moment.

\section{Market segmentation}

In this section, we still assume the presence of agents from both pools. Let us restrict our attention to a particular class of equilibria, namely segmented equilibria. The occurrence of market segmentation is not new to the literature (see Merton (1987) and Sharpe (1991) among others). Segmented-market versions of the CAPM are usually obtained imposing external constraints on the investment opportunity set. On the contrary, we aim at studying equilibria where segmentation arises endogenously in the model.

From now on we assume the presence of a single risky asset, i.e. Assumption 1 holds with $N=1$. We recall that this risky security has a per-share payoff $D_{1} \sim N\left(\mu_{1}, \sigma_{1}^{2}\right)$ and its exogenous supply is $S_{1}>0$. For the sake of clarity, we prefer to work with primary variables without using the market portfolio.

Definition 2 (Segmented equilibrium). A segmented equilibrium consists of a strictly positive price $\bar{q}_{1} \in \mathbb{R}_{+}$and allocations $\bar{\theta}^{j} \in \mathbb{R}, j \in \mathcal{I} \cup \mathcal{H}$, such that

(i) (Portfolio optimization) given $\bar{q}_{1}, \bar{\theta}^{j} \in \arg \max _{\theta^{j} \in \mathbb{R}} U^{j}\left(X^{j}\right), j \in \mathcal{I} \cup \mathcal{H}$, and $\bar{\theta}_{1}^{h}=0, h \in \mathcal{H}$;

(ii) (Market clearing) given $\bar{q}_{1}, \sum_{i=1}^{I} \bar{\theta}_{1}^{i}=S_{1}$.

Loosely speaking, a segmented equilibrium is a market equilibrium where for the CPT-MV agents it is optimal a totally risk-less investment. Therefore, the market clearing condition is satisfied through the aggregate demand of the EU traders. The reason for such a definition is to model an hypothetical economy whose agents can be divided into two groups, namely risky market participants and non-participants ${ }^{11}$. Thus, endogenously reaching a segmented equilibrium could explain high non-participation levels in the stock market as long as CPT-MV agents form the prevailing pool (see Haliassos and Bertaut (1995), Bertaut (1998) and Dimmock and Kouwenberg (2010)).

Before giving formal results, note that the Sharpe ratio of a portfolio that involves the single risky security can be computed as

$$
\xi_{1}=\frac{\mu_{1}-R_{f} q_{1}}{\sigma_{1}}, \quad q_{1} \in\left(0, \frac{\mu_{1}}{R_{f}}\right] .
$$

It follows that in equilibrium we necessarily have $\xi_{1} \in\left[0, \frac{\mu_{1}}{\sigma_{1}}\right)$. Recall that in the presence of exclusively CARA EU agents there exists an equilibrium with strictly positive prices if and only if $\Lambda<\frac{\mu_{1}}{\sigma_{1}^{2} S_{1}}$. Moreover, the equilibrium Sharpe ratio will be $\bar{\xi}_{1}=\Lambda \sigma_{1} S_{1}$, as we can deduce from (15).

\footnotetext{
${ }^{10}$ In the case of indifference with respect to her risky position, an ill-behaved trader could clear the market by acquiring the risky securities that the other participants are not willing to purchase. For example, this would make sense in a stock-exchange placing where unsold securities are bought by a single institution. On the other hand, it is hard to think of a portfolio manager or a household who is indifferent in bearing undefined risks. We will not deepen on this type of equilibria.

${ }^{11}$ We could use a more general notion of segmented equilibrium where the group of participants coincide with that of well-behaved traders and the group of non-participants contains the ill-behaved traders. Unfortunately, it would prevent from obtaining explicit expressions in our results.
} 
For the CPT-MV investors, we can exploit $\xi_{1}$ to write down an equivalent expression for $v^{h}$. In fact, we have

$$
v^{h}\left(\theta_{1}^{h}, \xi_{1}\right)=\int_{-\xi_{1}}^{+\infty} u^{h}\left(\left(t+\xi_{1}\right) \sigma_{1} \theta_{1}^{h}\right) \mathrm{d}\left[-w_{+}^{h}(1-\Phi(t))\right]+\int_{-\infty}^{-\xi_{1}} u^{h}\left(\left(t+\xi_{1}\right) \sigma_{1} \theta_{1}^{h}\right) \mathrm{d}\left[w_{-}^{h}(\Phi(t))\right],
$$

assuming that the previous integrals exist. Now, the definition of segmented equilibrium implies

$$
0 \in \arg \max _{\theta_{1} \geq 0} v^{h}\left(\theta_{1}, \bar{\xi}_{1}\right), \quad h=1, \ldots, H .
$$

In particular, if $\theta_{1}^{h}=0$ is not the unique maximum point for $v^{h}\left(\cdot, \bar{\xi}_{1}\right)$, we can impose the equilibrium allocation $\bar{\theta}_{1}^{h}=0$. Therefore, the last step to prove the existence of segmented equilibria is to find conditions that imply (32).

Lemma 4 (Sufficient conditions for (32)). For the h-th trader, each of the following conditions implies $(32)$ :

(i) (Linear LA) preferences are as in Lemma 1, (iii), with

$$
\lambda^{h} \geq \bar{\lambda} \equiv \frac{1+\sqrt{2 \pi} \Lambda \sigma_{1} e^{\Lambda^{2} \sigma_{1}^{2} / 2} \Phi\left(\Lambda \sigma_{1}\right)}{1-\sqrt{2 \pi} \Lambda \sigma_{1} e^{\Lambda^{2} \sigma_{1}^{2} / 2} \Phi\left(-\Lambda \sigma_{1}\right)}
$$

(ii) (Piecewise-power utility) preferences are as in Lemma 1, (ii), with $f^{h}(x)=x^{\alpha^{h}}$ and $F^{h}\left(\bar{\xi}_{1}\right) \leq 0$, where $F^{h}$ is defined in (25).

Proof. To prove (i) we follow the lines of Example 1. Adapting (16) with the parameters $\mu_{1}, \sigma_{1}$ and using the variables $\left(\theta_{1}^{h}, \xi_{1}\right)$, we can perform integration and obtain (33).

For (ii), it suffices to adapt (23) and (24) with the variables $\left(\theta_{1}^{h}, \xi_{1}\right)$ and note that the homogeneity in $\theta_{1}^{h}$ is preserved.

We incidentally observe that $\bar{\lambda}$ does not depend on $R_{f}$ and $\mu_{1}$, nor on $S_{1}$. Computing the firstorder derivatives, we see that $\bar{\lambda}$ is increasing in both $\Lambda$ and $\sigma_{1}$. Now, $\Lambda$ grows when there are less CARA EU traders (more generally, when a CARA coefficient increases). In such a situation, the aggregate demand of the pool $\mathcal{I}$ remains the same since it must clear the market, whereas the candidate equilibrium price $\bar{q}_{1}$ falls. This in turn attracts stock purchases and a CPT-MV agent stays out of the security market only if she has a higher loss-aversion coefficient. On the other hand, a rise in $\sigma_{1}$ means greater variance, which should in turn discourage risky investment. However, (33) imposes a stricter bound on the loss-aversion coefficient and this fact in counterintuitive. Finally, we note that an analytic (but not explicit) expression could be given for the well-behaved piecewise-exponential utility traders using (26), but it is not useful for our purposes.

Using the preceding lemma, we have the main result of this section.

Proposition 6 (Sufficient conditions for the existence of a segmented equilibrium). Let Assumptions 1-5 hold with $\Lambda<\frac{\mu_{1}}{\sigma_{1}^{2} S_{1}}$. Suppose that the preferences of each CPT-MV investor satisfy one of the conditions (i) or (ii) of Lemma 4. Then there exists a segmented equilibrium with $\bar{q}_{1}=\frac{1}{R_{f}}\left(\mu_{1}-\Lambda \sigma_{1}^{2} S_{1}\right)$ and $\bar{\theta}_{1}^{i}=\frac{\Lambda}{\lambda^{i}} S_{1}$.

Proof. The proposed equilibrium is the one attained when the market is populated by only CARA EU investors. Given such a $\bar{q}_{1}$, we compute $\bar{\xi}_{1}$ through $(30)$ and check that it implies null risky investment for every CPT-MV agent. But thanks to Lemma 4, condition (32) holds for all $h \in \mathcal{H}$ and we conclude.

\section{$5 \quad$ Segmented equilibrium with endogenous risk-free return}

A deep analysis of the market segmentation model introduced in the preceding section reveals a drawback. Suppose for a moment that the pool $\mathcal{I}$ of stock market participants is endowed with an extremely small percentage of the overall wealth of the economy, whereas the remaining wealth is shared among the members in $\mathcal{H}$. If a segmented equilibrium is attained, the price of the risky 
security (and its return) is exclusively determined by the joint effect of market parameters and EU agents' preferences (see the statement of Proposition 6). At the same time, the huge amount of money owned by the CPT-MV investors is totally conveyed in the risk-less asset market without affecting the risk-free return $R_{f}$. Not only, $R_{f}$ acts negatively (positively) on the equilibrium price (expected return), but as long as $R_{f}$ is exogenously given, $\bar{q}_{1}$ and $\bar{R}_{1}$ are not influenced. Instead, a more realistic scenario has to take into account the effect of massive risk-less investment. In particular, $R_{f}$ should decrease (increase) as money flows into (out of) the risk-free market.

We proceed to add this intuition to our model. Firstly, we remove Assumptions 1 and 2 and we replace it with the new hypothesis:

Assumption 6 (Market structure). There are 2 traded assets in a frictionless market without constraints.

- Asset 0 is the risk-free asset whose unit payoff is $\mu_{0}$ units of currency in every state of the world. Its exogenous supply is $S_{0}>0$.

- Asset 1 is the risky security whose per-share payoff is $D_{1} \sim N\left(\mu_{1}, \sigma_{1}^{2}\right)$. Its exogenous supply is $S_{1}>0$.

Assumption 7 (Investor endowments). Each investor receives an initial wealth $W^{j} \geq 0, j \in \mathcal{I} \cup \mathcal{H}$, in units of currency.

Observe that the risk-less asset is no more in elastic supply, as it happens in actual Government or corporate bond markets. Moreover, for convenience we can think of $W^{j}$ as the savings amount of the $j$-th agent that must be divided among risky and risk-less investment.

We recover the notation from the previous sections: $q=\left(q_{0}, q_{1}\right)$ is the price vector, where also $q_{0}$ must be determined endogenously. Consequently, the expected return $R_{0}=\mu_{0} / q_{0}$ is endogenous as well as $R_{1}=\mu_{1} / q_{1}$. We restrict our attention to segmented equilibria.

Definition 3 (Segmented equilibrium with endogenous risk-free return). A segmented equilibrium is a vector of strictly positive prices $\bar{q}=\left(\bar{q}_{0}, \bar{q}_{1}\right) \in \mathbb{R}_{++}^{2}$ and allocations $\bar{\theta}^{j} \in \mathbb{R}, j \in \mathcal{I} \cup \mathcal{H}$, such that

(i) (Portfolio optimization) given $\bar{q}, \bar{\theta}^{j} \in \arg \max _{\theta^{j} \in \mathbb{R}} U^{j}\left(X^{j}\right), j \in \mathcal{I} \cup \mathcal{H}$, and $\bar{\theta}_{1}^{h}=0, h \in \mathcal{H}$;

(ii) (Market clearing) given $\bar{q}, \sum_{i=1}^{I} \bar{\theta}_{n}^{i}+\sum_{h=1}^{H} \bar{\theta}_{n}^{h}=S_{n}, n=0,1$.

We immediately observe that the standard market clearing condition (ii) can be written in a more intuitive way. Recall the budget constraints, namely

$$
W^{j}=q_{0} \theta_{0}^{j}+q_{1} \theta_{1}^{j}, \quad j \in \mathcal{I} \cup \mathcal{H},
$$

and denote

$$
W^{\mathcal{I}}:=\sum_{i=1}^{I} W^{i}, \quad W^{\mathcal{H}}:=\sum_{h=1}^{H} W^{h}, \quad W:=W^{\mathcal{I}}+W^{\mathcal{H}},
$$

the total initial wealths of the two pools and the aggregate wealth of the economy respectively. Summing the budget constraints over $i \in \mathcal{I}$ and $h \in \mathcal{H}$ and using the segmented equilibrium condition $\bar{\theta}_{1}^{h}=0, h \in \mathcal{H}$, we obtain the equivalent market clearing condition

$$
\left\{\begin{array}{l}
\bar{q}_{0} S_{0}+\bar{q}_{1} S_{1}=W \\
\sum_{i=1}^{I} \bar{\theta}_{1}^{i}=S_{1}
\end{array}\right.
$$

The first equation in (34) is nothing but the budget constraint of the economy, whereas the second equation means that EU agents clear the market for the risky asset. Looking for equilibria, we can carefully go through our analysis replacing $R_{f}$ (or $1+r$ ) with the risk-less return $R_{0}$; however, this time $q_{0}$ must be treated as a variable. An important thing to note is that the reference points $R P^{h}$ of our CPT-MV traders are no more well defined. So far, we fixed $R P^{h}=W^{h} R_{f}$, and this was sensible as long as $R_{f}$ was an exogenous parameter. From now on, we set $R P^{h}=W^{h} R_{0}$ as the endogenous reference point. As noted in Köszegi and Rabin (2006), a good reason for an endogenous $\mathrm{RP}$ is that the economy itself can determine the RPs through the achievement of personal equilibria. In particular, the RP can be seen as a rational expectation about outcomes that must be consistent 
with optimizing behavior, given that expectation. In our world, the RP becomes the risk-free return adjusted initial wealth, given that return. Note that $R_{0}$ will be the result of the optimal choices by every agent. Therefore, in our model the $R P \mathrm{~s}$ will be determined by the whole economy in a general equilibrium. In the next proposition we characterize a segmented equilibrium and we provide sufficient conditions for its existence.

Proposition 7 (Segmented equilibrium with endogenous risk-free return). Let Assumptions 3-7 hold.

(i) (Necessary condition) If a segmented equilibrium exists, then the equilibrium prices are

$$
\bar{q}_{0}=\frac{\mu_{0} W}{\mu_{0} S_{0}+\nu_{1} S_{1}}, \quad \bar{q}_{1}=\frac{\nu_{1} W}{\mu_{0} S_{0}+\nu_{1} S_{1}},
$$

and the equilibrium allocation are

$$
\begin{array}{lll}
\bar{\theta}_{0}^{i}=\frac{\lambda^{i} W^{i}\left(\mu_{0} S_{0}+\nu_{1} S_{1}\right)-\nu_{1} S_{1} \Lambda W}{\lambda^{i} \mu_{0} W}, & \bar{\theta}_{1}^{i}=\frac{\Lambda}{\lambda^{i}} S_{1}, \\
\bar{\theta}_{0}^{h}=\frac{W^{h}\left(\mu_{0} S_{0}+\nu_{1} S_{1}\right)}{\mu_{0} W}, & \bar{\theta}_{1}^{h}=0,
\end{array}
$$

where $\nu_{1}:=\mu_{1}-\Lambda \sigma_{1}^{2} S_{1}$.

(ii) (Sufficient condition) If $\Lambda<\frac{\mu_{1}}{\sigma_{1}^{2} S_{1}}$ and the preferences of each CPT-MV investor satisfy one of the conditions ( $i$ ) or (ii) of Lemma 4 , then an equilibrium exists (and it is characterized by (35) and (36)).

Proof. For (i), having assumed EU CARA traders we know that utility maximization implies

$$
\theta_{1}^{i \star}=\frac{\mu_{1}-\mu_{0} \frac{q_{1}}{q_{0}}}{\lambda^{i} \sigma_{1}^{2}} .
$$

Summing over $i \in \mathcal{I}$ to satisfy the market clearing condition (34), we see that in equilibrium it necessarily holds

$$
\bar{q}_{1}=\frac{\nu_{1}}{\mu_{0}} \bar{q}_{0}
$$

Solving the linear system

$$
\left\{\begin{array}{l}
\bar{q}_{0} S_{0}+\bar{q}_{1} S_{1}=W, \\
\bar{q}_{1}=\frac{\nu_{1}}{\mu_{0}} \bar{q}_{0},
\end{array}\right.
$$

yields the equilibrium prices. Finally, equilibrium allocation can be obtained via (37) and the personal budget constraints. For (ii), it suffices to adapt the proof of Proposition 6.

Thanks to (35) we can compute the expected equilibrium returns:

$$
\bar{R}_{0}=\frac{\mu_{0} S_{0}+\nu_{1} S_{1}}{W}, \quad \bar{R}_{1}=\frac{\mu_{1}}{\nu_{1}} \frac{\left(\mu_{0} S_{0}+\nu_{1} S_{1}\right)}{W} .
$$

Note that if an equilibrium exists, then $\bar{R}_{1}>\bar{R}_{0}$, since $\mu_{1}>\nu_{1}{ }^{12}$. Moreover,

$$
\bar{R}_{0} \geq 1 \Longleftrightarrow\left(\mu_{0} S_{0}+\mu_{1} S_{1}\right)-\Lambda\left(\sigma_{1} S_{1}\right)^{2} \geq W .
$$

In other words, the risk-free interest rate is positive if and only if the overall expected payoff of the market $\left(\mu_{0} S_{0}+\mu_{1} S_{1}\right)$ corrected by the product between the market price of risk $\Lambda$ and the total variance of the market $\left(\sigma_{1} S_{1}\right)^{2}$ is greater than the aggregate savings of the economy. Hence, we can not a priori exclude the existence of equilibria with negative risk-less interest rate. For the CPT-MV investors, $\bar{R}_{0}<1$ implies a RP lower than the initial wealth. This amounts to say that these agents expect (and actually realize) a decrease of their savings ${ }^{13}$.

Now we perform a comparative statics analysis. Computing first-order derivatives, we find the next result (for convenience we suppressed the bar superscript).

\footnotetext{
${ }^{12}$ We are not able to say much more about the equity premium $\bar{R}_{1}-\bar{R}_{0}$. In any case, our equilibria can sustain the empirically observed "equity premium puzzle"; see Mehra and Prescott (1985).

${ }^{13}$ With the exception of the Swedish case in July 2009, when the Riksbank (the Swedish central bank) used negative interest rate on deposits at $-25 \mathrm{bp}$, nominal interest rates never become negative. However, real interest rates often goes below zero, as it happened for Italian BoT (Italian treasury bonds) in September 2008 and for U.S. Tips (Treasury inflation protected securities) in October 2010. Interpreting $\bar{R}_{0}$ and $\bar{R}_{1}$ as real expected returns, we can incorporate inflation in our model.
} 


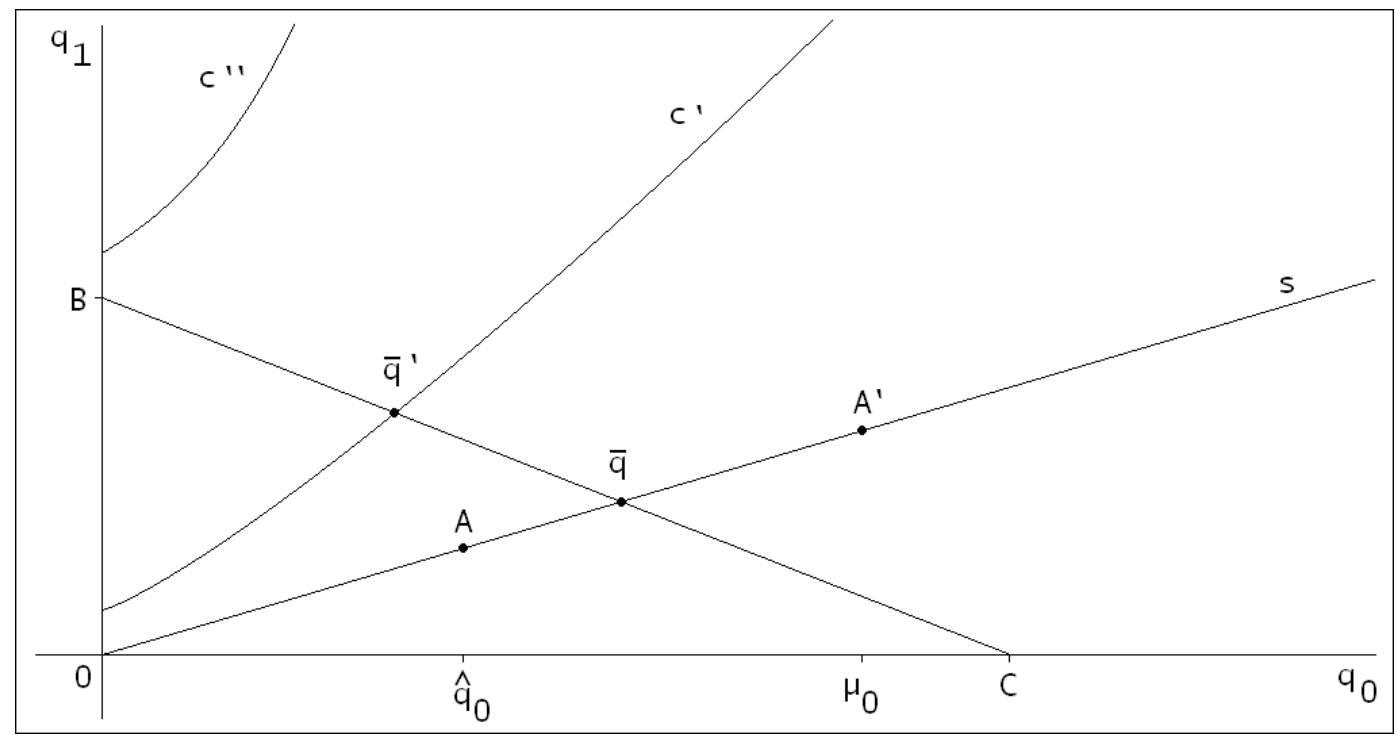

Figure 4: The figure shows some possible scenarios for a segmented-market model with endogenous risk-free return. On a $\left(q_{0}, q_{1}\right)$ plane, we depicted the economy budget constraint $\overline{B C}$ and the equilibrium line $s$ given by (38) in the CARA EU case. The intersection point $\bar{q}$ is the unique equilibrium. Removing Assumption 5, we could find the candidate equilibrium curve $c^{\prime}$ that implies existence of a unique equilibrium $\bar{q}^{\prime}$. Otherwise, the curve $c^{\prime \prime}$ does not lead to an equilibrium. Finally, restoring Assumption 5 and removing Assumption 7 , we identify a segment $\overline{A A^{\prime}}$ that specifies an attainable subset of equilibria.

Corollary 1 (Comparative statics on equilibrium returns). The following relations hold:

$$
\begin{aligned}
\frac{\partial R_{1}}{\partial S_{0}}>\frac{\partial R_{0}}{\partial S_{0}}>0, & \frac{\partial R_{1}}{\partial S_{1}}>\frac{\partial R_{0}}{\partial S_{1}}, & \frac{\partial R_{1}}{\partial \mu_{0}}>\frac{\partial R_{0}}{\partial \mu_{0}}>0, & \frac{\partial R_{1}}{\partial \mu_{1}}<\frac{\partial R_{0}}{\partial \mu_{1}} \\
\frac{\partial R_{1}}{\partial \sigma_{1}}>0>\frac{\partial R_{0}}{\partial \sigma_{1}}, & \frac{\partial R_{1}}{\partial \Lambda}>0>\frac{\partial R_{0}}{\partial \Lambda}, & \frac{\partial R_{1}}{\partial W^{\mathcal{I}}}<\frac{\partial R_{0}}{\partial W^{\mathcal{I}}}<0, & \frac{\partial R_{1}}{\partial W^{\mathcal{H}}}<\frac{\partial R_{0}}{\partial W^{\mathcal{H}}}<0 .
\end{aligned}
$$

Observe that we are not able to assess the sign of certain derivatives as they depend on mixtures of the model's parameters. In any case, the preceding inequalities generally confirm economic intuition. It is worthwhile saying that an increase in one investor's wealth produces a fall in both expected returns, no matter which pool the trader comes from. Not only, $R_{1}$ is always more affected than $R_{0}$ and this is an unpleasant prediction of this model. In fact, we would expect that raising $W^{\mathcal{H}}$ causes a greater drop in $R_{0}$ than in $R_{1}$. The source of this flaw lies in the CARA hypotheses over EU agents. As it appears clear from equation (37), the optimal demand of EU traders does not depend on the wealth $W^{i}$. The same is true for the relation (38) between $q_{0}$ and $q_{1}$ that must hold in equilibrium. Consequently, the equilibrium prices (35) depend linearly on $W$ and this ultimately produces the drawback.

Interestingly, this model fits well for a graphical analysis; see Figure 4. Restricting to positive prices, on a $\left(q_{0}, q_{1}\right)$ plane we can depict the economy budget constraint (i.e. the first equation in (39)) as an open segment $\overline{B C}$. Observe that an equilibrium (if it exists) must lie on $\overline{B C}$. If Assumption 5 on CARA EU traders holds, then we find (38), which describes a positively inclined open half-line $s$. The intersection point between the two lines gives the unique equilibrium price vector $\bar{q}$.

We complete our analysis suggesting two possible extensions of the model. First, if we remove Assumption 5 things become quite involved. Assuming sufficient regularity, for given initial wealth $W^{i}$ we could find the amount of money invested by the $i$-th agent in the risky asset and suppose that it is given by the function $\eta^{i}\left(q_{0}, q_{1}, W^{i}\right)$, where intuition suggests $\frac{\partial \eta^{i}}{\partial q_{0}}>0, \frac{\partial \eta^{i}}{\partial q_{1}}<0, \frac{\partial \eta^{i}}{\partial W^{i}}>0$. Now, summation over $i \in \mathcal{I}$ yields the market clearing condition for the risky asset, namely

$$
\sum_{i=1}^{I} \frac{\eta^{i}\left(\bar{q}_{0}, \bar{q}_{1}, W^{i}\right)}{\bar{q}_{1}}=S_{1} .
$$


The previous equation implicitly defines a function $q_{1}\left(q_{0}\right)$ which describes a locus of candidate equilibrium price vectors. Generally, it will be a regular curve on the $\left(q_{0}, q_{1}\right)$ plane and using the implicit function theorem we get

$$
\frac{d q_{1}}{d q_{0}}=\frac{\sum_{i=1}^{I} \frac{\partial \eta^{i}}{\partial q_{0}}}{S_{1}-\sum_{i=1}^{I} \frac{\partial \eta^{i}}{\partial q_{1}}} .
$$

Suppose the signs of the partial derivatives of $\eta^{i}$ are those we assumed before. In such case, we see that if $q_{1}\left(q_{0}\right)$ intersects the economy budget constraint, then it necessarily produces an upward crossing. The (possible) intersection points yield equilibrium price vectors, though they will be generally different from the one described in Proposition 7 (see the curve $c^{\prime}$ in Figure 4 and the relative equilibrium $\bar{q}^{\prime}$ ). On the other hand, we can not assure the existence of a crossing point. This last situation is represented by the curve $c^{\prime \prime}$ in Figure 4. Not surprisingly, the derivatives of $\eta^{i}$ can have different signs with respect to the aforementioned case; see Ingersoll (1987). Therefore, it remains an open question whether equilibria exist and how they can be found.

Suppose instead to remove Assumption 7 on the exogenous endowments and restore Assumption 5 concerning CARA EU agents. Analytically, this means that (38) holds while the economy budget constraint becomes a redundant equation. A look at Figure 4 shows that we have an entire line of equilibria since each point on $s$ can sustain an equilibrium. The problem now consists in finding the equilibrium that will be attained. At first, we can put some natural bounds on $\bar{q}_{0}$. It seems sensible to choose $q_{0} \leq \mu_{0}$ in order to have an equilibrium with positive risk-free interest rate. Then, we could suppose that the issuer of the risk-less asset only accepts to place its securities at a sufficiently high price, i.e. $q_{0} \geq \hat{q}_{0}$ for some $\hat{q}_{0}>0{ }^{14}$. The set of equilibria is therefore reduced to the segment $\overline{A A^{\prime}}$ in Figure 4. It is also clear that for the CPT-MV agents there is indifference since once a segmented equilibrium is reached, we have $v^{h}\left(X^{h}\right)=0$ no matter which equilibrium is selected. In conclusion, the equilibrium pair $\left(\bar{q}_{0}, \bar{q}_{1}\right)$ depends on the "market power" of the participants. If EU traders are the prevailing force, then the attained equilibrium will be close to $\left(\hat{q}_{0}, \frac{\nu_{1}}{\mu_{0}} \hat{q}_{0}\right)$, located at $A$ in Figure 4. In fact, for any pair $\left(q_{0}, q_{1}\right)$ satisfying $(38)$, we have

$$
U^{i}\left(X^{i}\right)=-\exp \left\{-\frac{\mu_{0} W^{i} \lambda^{i}}{q_{0}}-\frac{1}{2} \Lambda^{2} \sigma_{1}^{2} S_{1}^{2}\right\},
$$

which is monotone decreasing in $q_{0}$ and reaches its maximum for $q_{0}=\hat{q}_{0}$. On the contrary, as the power of the bond issuer increases, the equilibrium will move toward $\left(\mu_{0}, \nu_{1}\right)$, i.e. the point $A^{\prime}$ in Figure 4.

\section{Conclusions}

Including heterogeneous agents in financial market models adds realism to the description of actual economies. At the same time, research of equilibria becomes more sophisticated and (strong) assumptions are needed to retain analytical tractability. Even in an extremely simple setting of one risk-free and one risky asset, the existence of an equilibrium with positive asset prices is not guaranteed. This drawback was already known in the presence of solely expected utility maximizers and it equally arises with non-expected utility paradigms.

Assuming expected utility and cumulative prospect theory preferences, we find that the classical CAPM provides a necessary basis for heterogeneous agents models as well as it happened in homogeneously populated economies. Depending on the particular choices of the utility functions, it is possible to give necessary and/or sufficient conditions for the existence of an equilibrium. Focusing on segmented equilibria, we provide an intuitive explanation for the high non-participation levels of households in the stock markets, as widely documented in advanced economies.

Furthermore, we leave a number of open questions for future research. First, we wish to find conditions that assure equilibria with positive prices when there is more than one risky security. Second, we have seen that removing the constant absolute risk aversion hypothesis over the pool of expected utility maximizers strongly involves the model; in particular, an equilibrium needs not to exist or there can be plenty of equilibria. At last, we aim at introducing some selection criteria when more than one equilibrium is attainable.

\footnotetext{
${ }^{14}$ We can think to the issuer as a firm that places its bonds with a bid auction where the initial price is set to $\hat{q}_{0}$.
} 


\section{References}

[1] M. Allingham (1991): Existence theorems in the capital asset pricing model. Econometrica, 59, 1169-1174.

[2] N. Barberis and M. Huang (2008): Stocks as lotteries: The implications of probability weighting for security prices. The American Economic Review, 98(5), 2066-2100.

[3] N. Barberis, M. Huang and T. Santos (2001): Prospect theory and asset prices. Quarterly Journal of Economics, 116(1), 1-53.

[4] C. Bernard and M. Ghossoub (2010): Static portfolio choice under cumulative prospect theory. Mathematics and financial economics, 2(4), 277-306.

[5] C.C. Bertaut (1998): Stockholding behavior of U.S. households: Evidence from the 1983-1989 Survey of Consumer Finances. The Review of Economics and Statistics, 80(2), 263-275.

[6] D. A. Chapman and V. Polkovnichenko (2009): First-order risk aversion, heterogeneity, and asset market outcomes. Journal of Finance, 64(4), 1863-1887.

[7] E. G. De Giorgi, T. Hens and H. Levy (2011): CAPM equilibria with prospect theory preferences. Working Paper, available at http://ssrn.com/abstract $=420184$.

[8] E. G. De Giorgi, T. Hens and M. O. Rieger (2010): Financial market equilibria with cumulative prospect theory. Journal of Mathematical Economics, 46(5), 633-651.

[9] S.G. Dimmock and R. Kouwenberg (2010): Loss-aversion and household portfolio choice. Journal of Empirical Finance, 17, 441-459.

[10] I. Fortin and J. Hlouskova (2011): Optimal asset allocation under linear loss aversion. Journal of Banking \& Finance, 35, 2974-2990.

[11] M. Haliassos and C.C. Bertaut (1995): Why do so few hold stocks? The Economic Journal, 105, 1110-1129.

[12] X.D. He and X.Y. Zhou (2011): Portfolio choice under cumulative prospect theory: An analytical treatment. Management Science, 57(2), 315-331.

[13] S. Hwang and S.E. Satchell (2010): How loss averse are investors in financial markets? Journal of Banking \& Finance, 34, 2425-2438.

[14] J.E. Ingersoll (1987): Theory of Financial Decision Making. Rowman and Littlefield.

[15] J.E. Ingersoll (2008): Non-monotonicity of the Tversky-Kahneman probability-weighting function: A cautionary note. European Financial Management, 14(3), 385-390.

[16] H. Jin and X.Y. Zhou (2008): Behavioral portfolio selection in continuous time. Mathematical Finance, 18, 385-426.

[17] D. Kahneman and A. Tversky (1979): Prospect theory: an analysis of decision under risk. Econometrica, 47(2), 263-291.

[18] V. Kobberling and P. Wakker (2006): An index of loss aversion. Journal of Economic Theory, $122(1), 119-131$.

[19] B. Köszegi and M. Rabin (2006): A model of reference-dependent preferences. The Quarterly Journal of Economics, 71(4), 1133-1165.

[20] H. Levy (1978): Equilibrium in an imperfect market: a constraint on the number of securities in the portfolio. The American Economic Review, 68, 643-658.

[21] H. Levy (2010): The CAPM is alive and well: A review and synthesis. European Financial Management, 16(1), 43-71. 
[22] M. Levy (2007): Conditions for a CAPM equilibrium with positive prices. Journal of Economic Theory, 137, 404-415.

[23] J. Lintner (1965): The valuation of risky assets and the selection of risky investments in stock portfolios and capital budgets. Review of Economics and Statistics, 47(1), 13-37.

[24] H. Markowitz (1990): Risk adjustment. Journal of Accounting, Auditing E3 Finance, 213-225.

[25] R. Mehra and E.C. Prescott (1985): The equity premium puzzle. Journal of Monetary Economics, 21, 145-161.

[26] R.C. Merton (1987): A simple model of capital market equilibrium with incomplete information. The Journal of Finance, 42, 483-510.

[27] J. Mossin (1966): Equilibrium in a capital asset market. Econometrica, 34(4), 768-783.

[28] L.T. Nielsen (1988): Uniqueness of equilibrium in the classical capital asset pricing model. Journal of Financial and Quantitative Analysis, 23(3), 329-336.

[29] L.T. Nielsen (1992): Positive prices in CAPM. The Journal of Finance, 47, 791-808.

[30] U. Schmidt and H. Zank (2005): What is loss aversion? The Journal of Risk and Uncertainty, $30: 2,157-167$.

[31] W.F. Sharpe (1964): Capital asset prices: A theory of market equilibrium under conditions of risk. The Journal of Finance, 19(3), 425-442.

[32] W.F. Sharpe (1991): Capital asset prices with and without negative holdings. The Journal of Finance, 46, 489-509.

[33] J. Tobin (1958): Liquidity preference as Behaviour towards risk. Review of Economic Studies, $25(2), 65-86$.

[34] A. Tversky and D. Kahneman (1992): Advances in prospect theory: Cumulative representation of uncertainty. Journal of Risk and Uncertainty, 5, 297-323.

[35] M. E. Yaari (1987): The Dual Theory of Choice under Risk. Econometrica, 55(1), 95-115. 